\title{
The Interpretation of Certain Norms of the General Administrative Code of Georgia and Law of Georgia on Police
}

\author{
Kakha Kurashvili \\ Doctor of Law, Caucasus's International University, Assistant-Professor
}

ARTICLE INFO

Article History:

Received 6.11.2021

Accepted 19.11.2021

Published 20.12.2021

Keywords:

Governance,

Body,

Offense

\section{ABSTRACT}

This article is related to issues of interpretation of certain norms defined under General Administrative Code of Georgia and Law of Georgia on Police. In particular, Article 3 of the General Administrative Code of Georgia regulates the scope of this code. However, provision of the Article 4 does not contain any reference to the administrative offenses committed by the police and other administrative bodies, what in specific cases may lead to ambiguity in regards the scope of this code - as subject required by the General Administrative Code of Georgia and Administrative Offenses Code of Georgia, in both cases is an authorized administrative body (officials).

Responding to administrative offenses by police is an important part of the activities carried out by the state authority (police). Therefore, Law of Georgia on Police distinguishes preventive function of the police from function of responding to offense. Also, the Article 5 of the law defines legal grounds for police activities, however this article does not contain specific references to Administrative Offenses Code of Georgia what can be deemed as legislative shortcoming.

Taking into consideration the above-mentioned, in order to clarify the law and to achieve objective goal of the legal norm, below listed terms shall be added to 1. General Administrative Code of Georgia, Section 4, Article 3, and 2. Law of Georgia on Police, Article 5. 


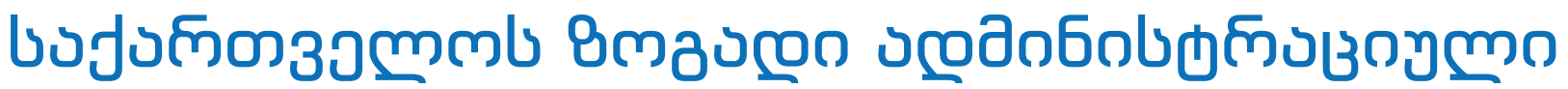

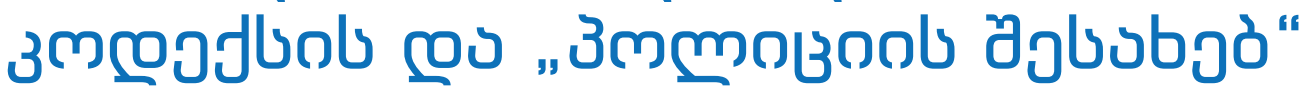

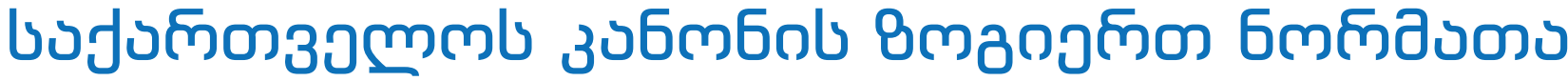

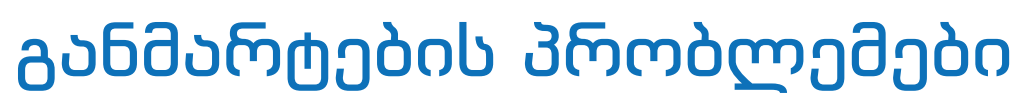

\author{
з

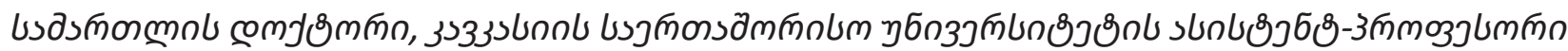

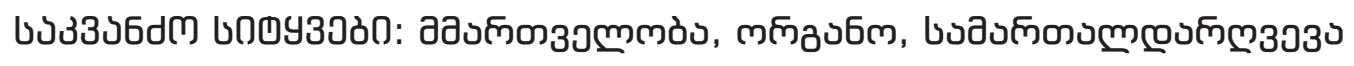

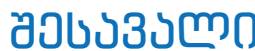

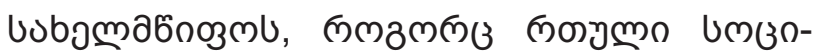

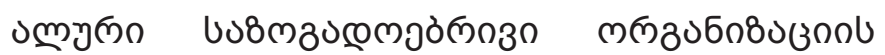

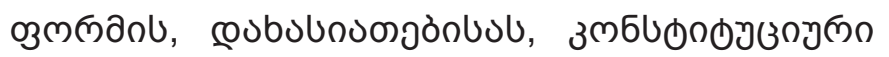

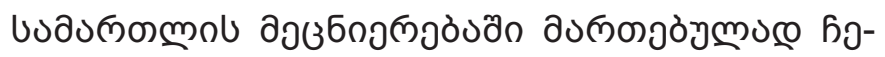

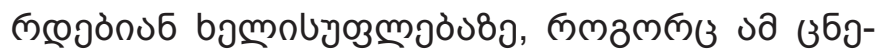

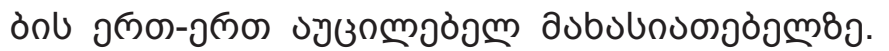

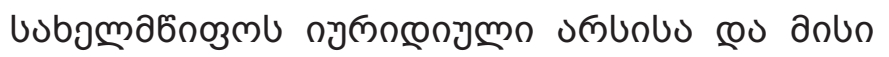

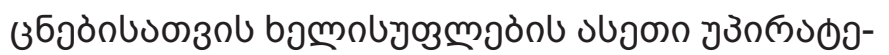

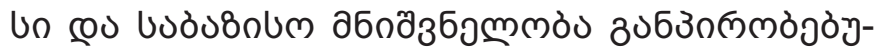

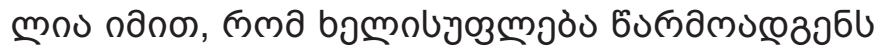

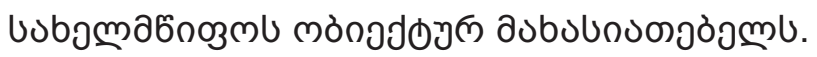

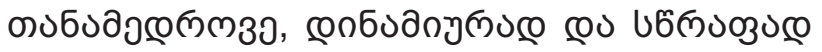

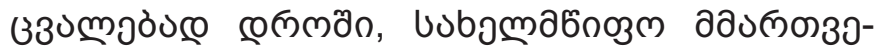

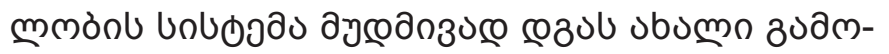

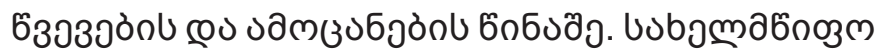

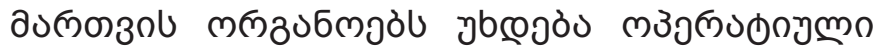

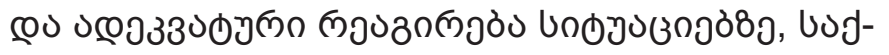

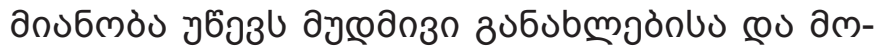

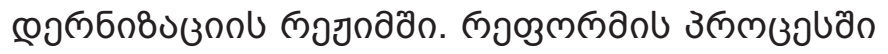

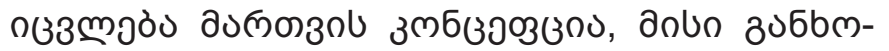

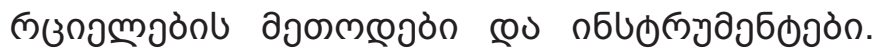

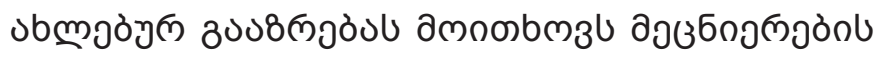

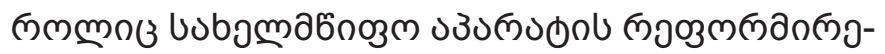

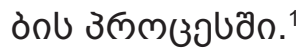

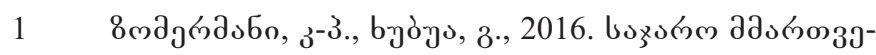

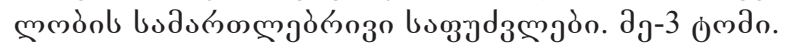

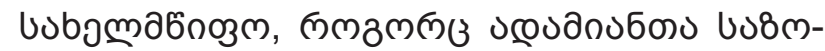

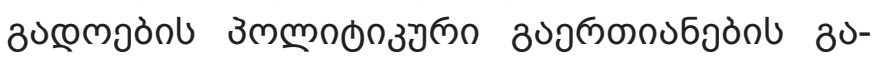

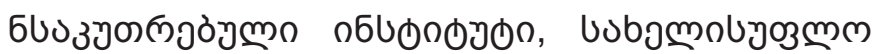

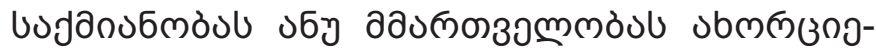

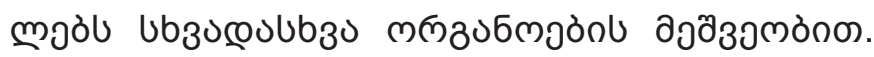

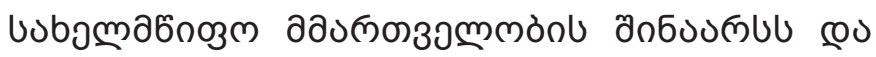

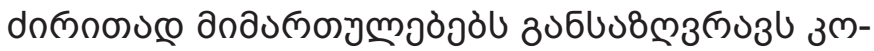

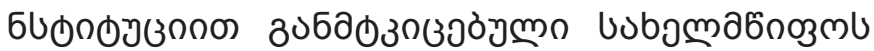

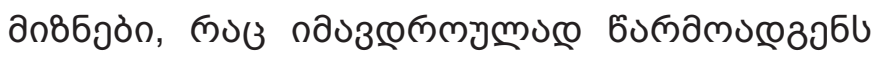

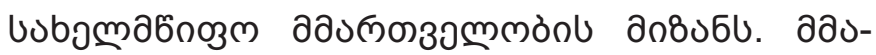

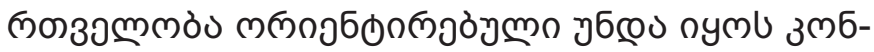

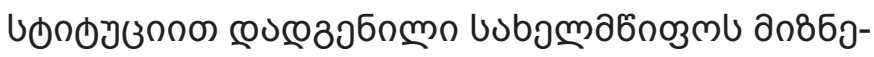

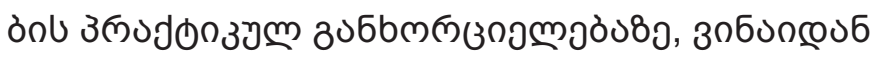

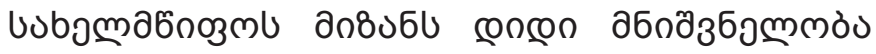

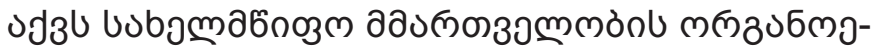

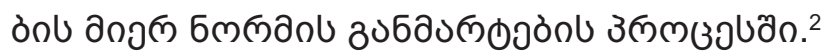

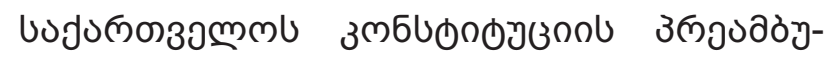

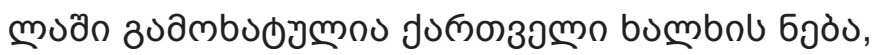

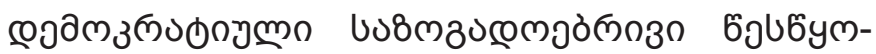

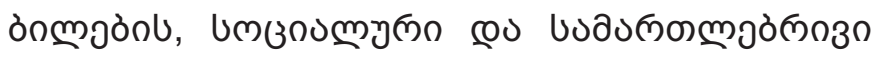

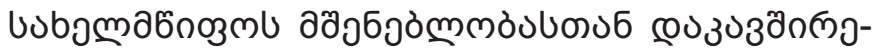

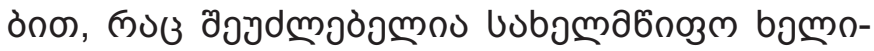

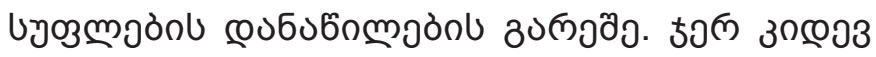

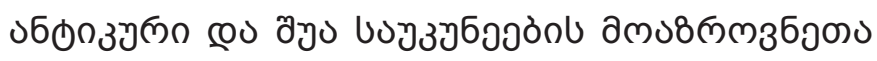

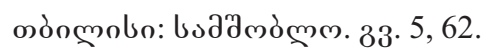

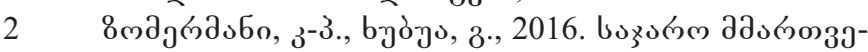

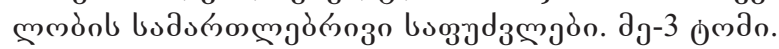

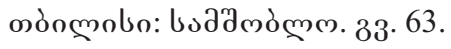




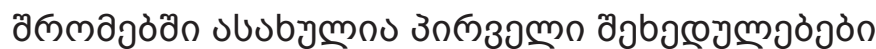

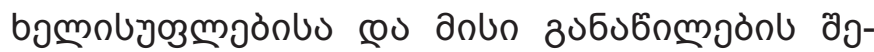

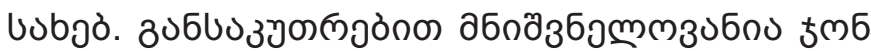

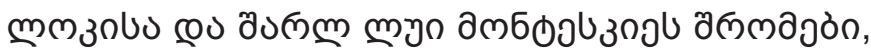

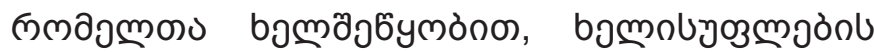

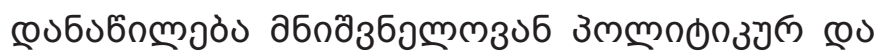

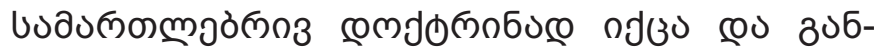

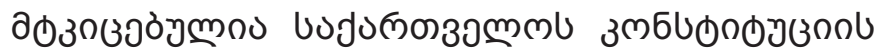

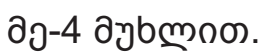

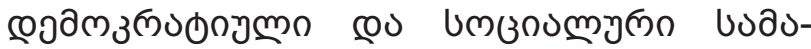

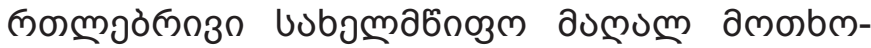

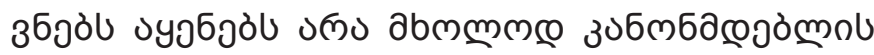

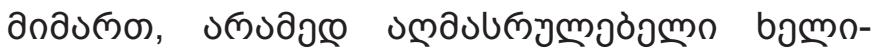

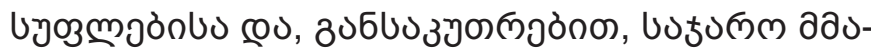

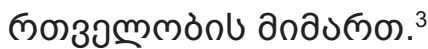

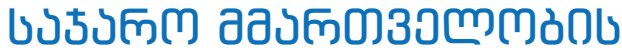

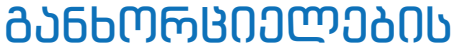

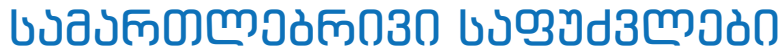

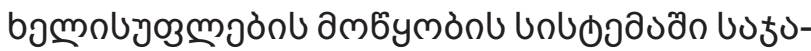

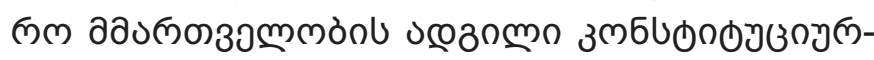

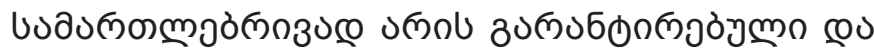

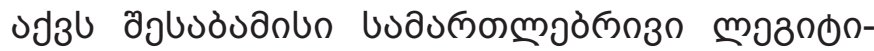

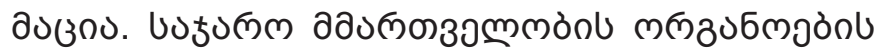

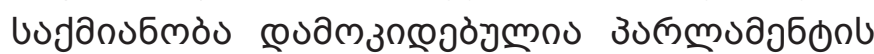

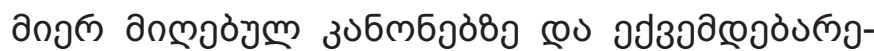

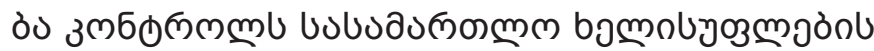

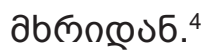

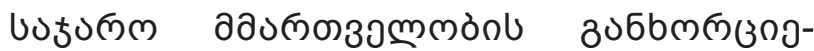

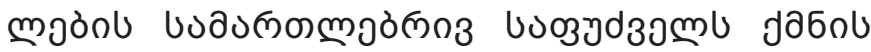

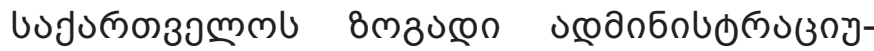

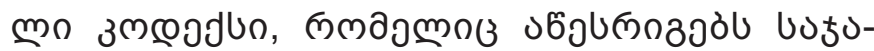

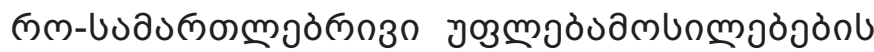

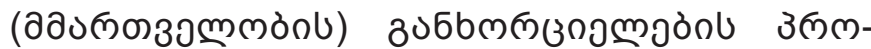
(304U

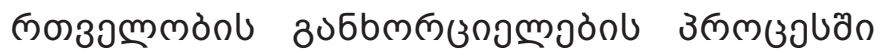

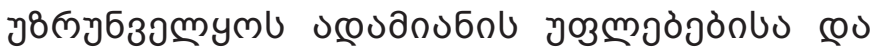

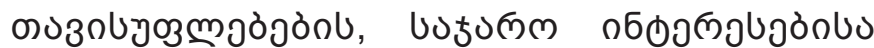

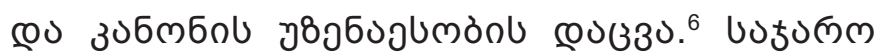

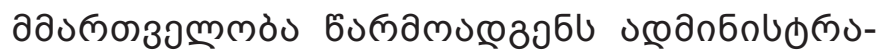

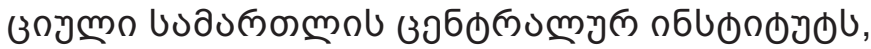

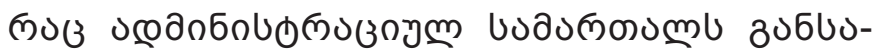

of $39 \cdot 33 \cdot 18$.

คป $39 \cdot 33 \cdot 107$

of39. $33 \cdot 108$

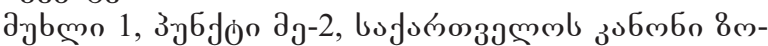

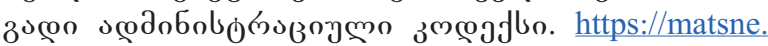
gov.ge/document/view $/ 16270$ ?publication $=36$

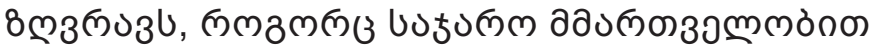
৩uasलnsmu.

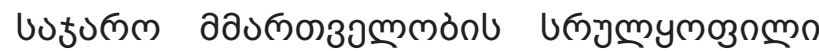

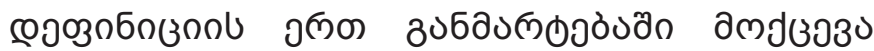

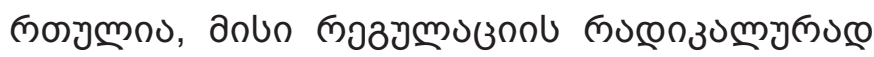

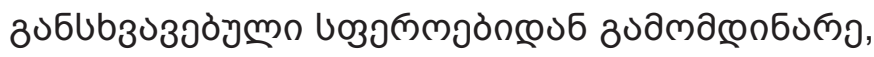

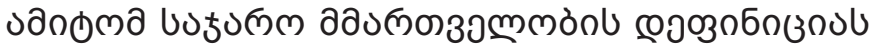

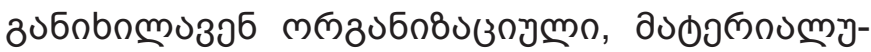

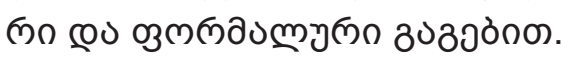

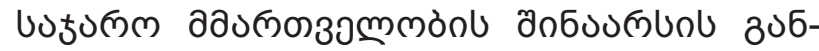

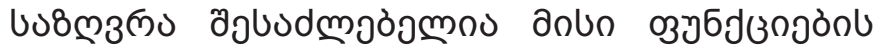

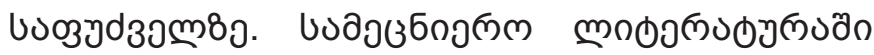

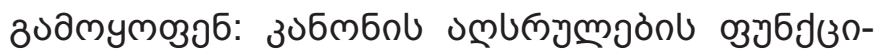

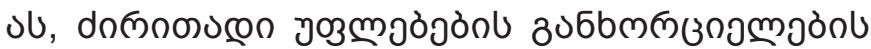

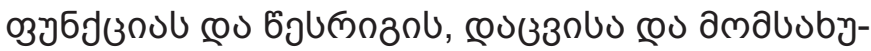

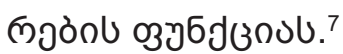

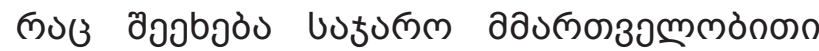

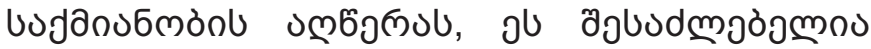

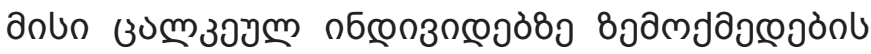

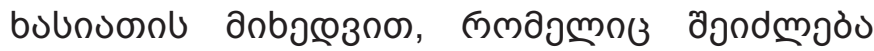

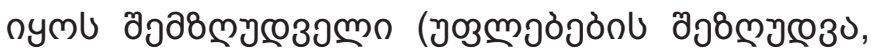

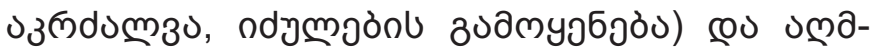

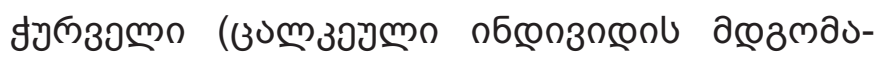

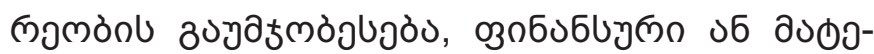

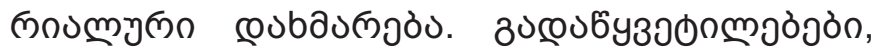

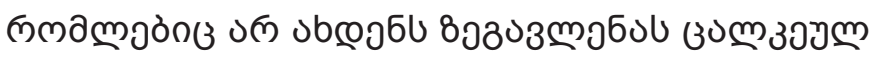

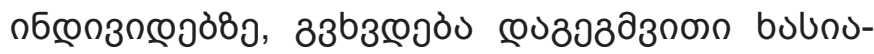

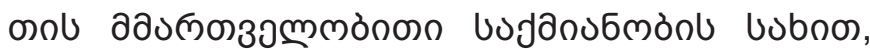

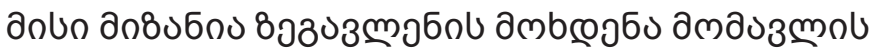
дмзмлбјठ8)).

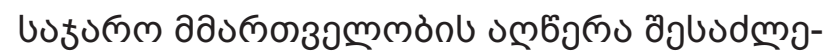

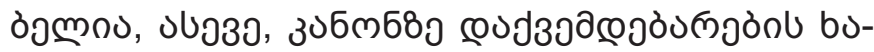

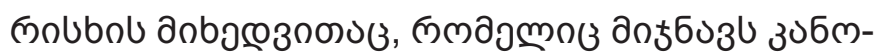

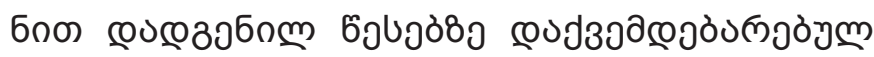

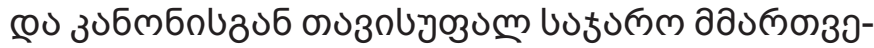

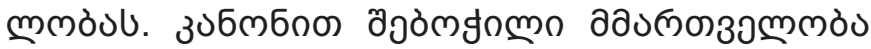

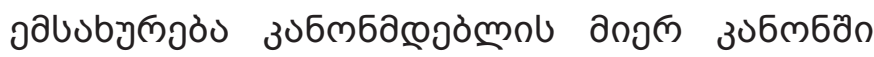

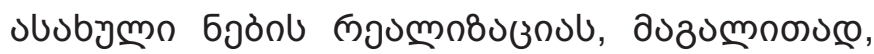

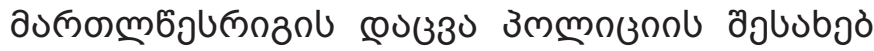

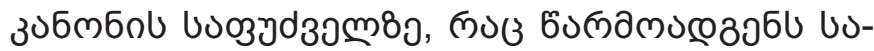

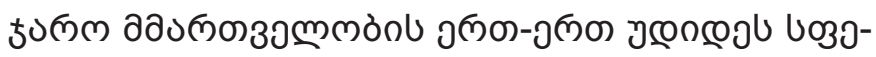
लmए. 8

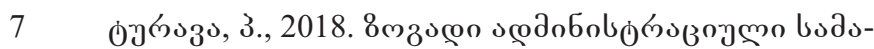

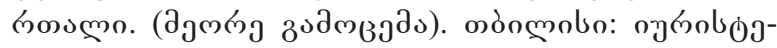
ònb badyox́. $33.13-14,16$.

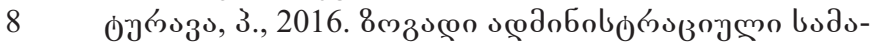

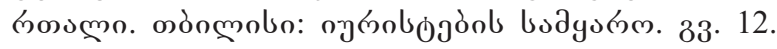

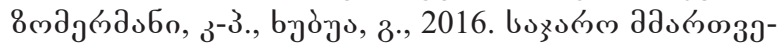

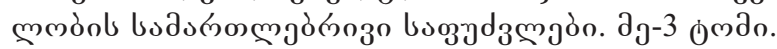

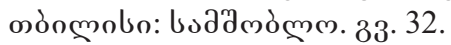




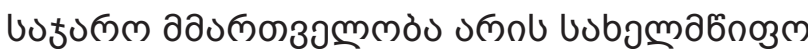

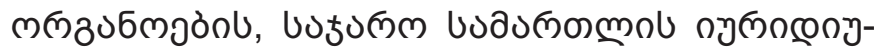

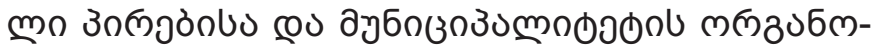

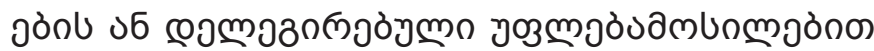

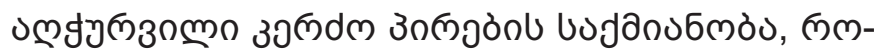

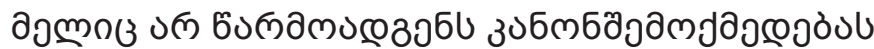

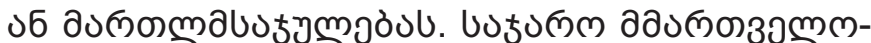

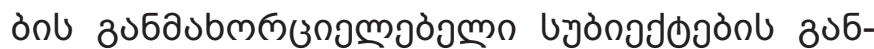

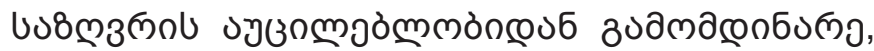

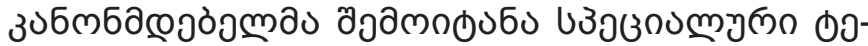

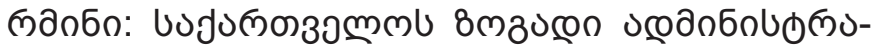

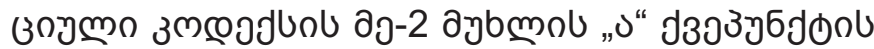

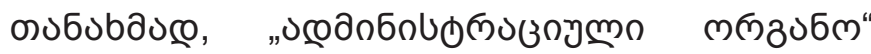

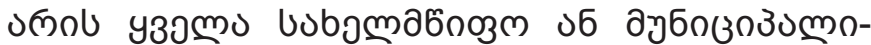

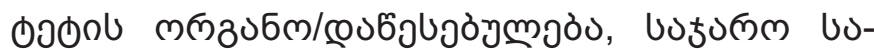

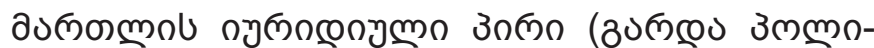

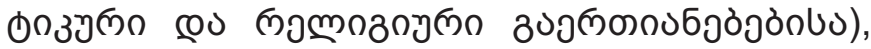

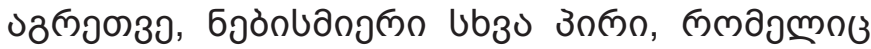

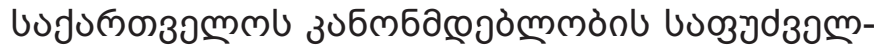

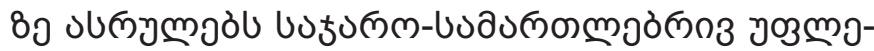

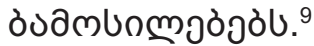

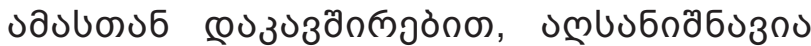

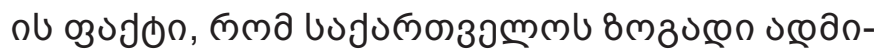

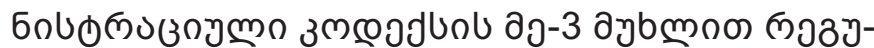

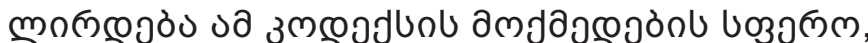

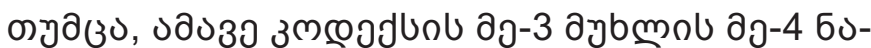

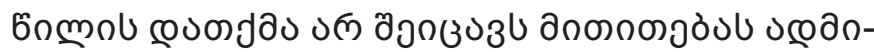

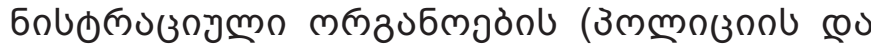

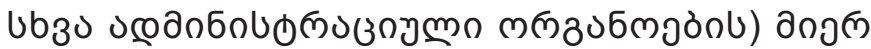

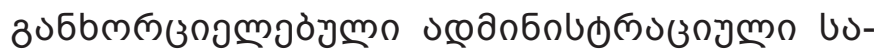

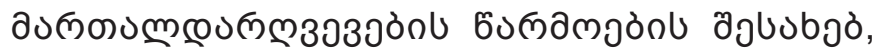

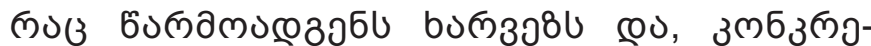

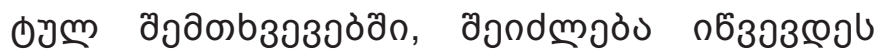

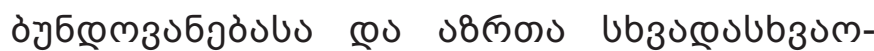

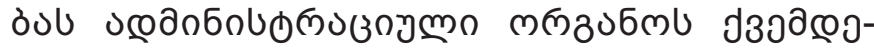

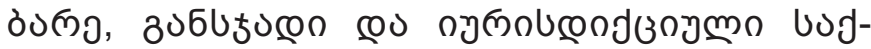

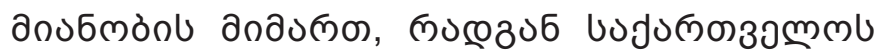

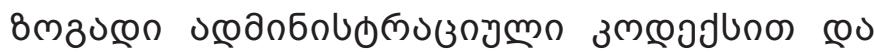

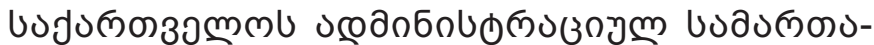

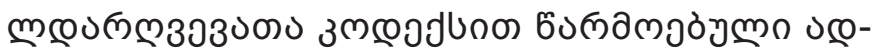

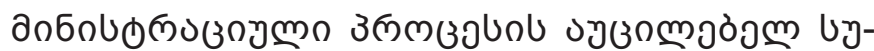

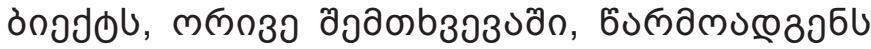

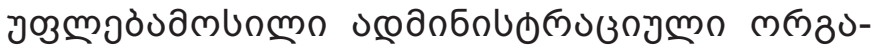
бм (๘)бақод்

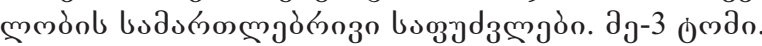

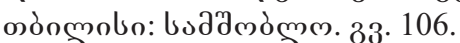

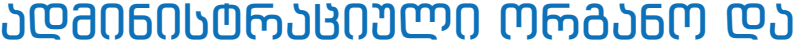 ง}

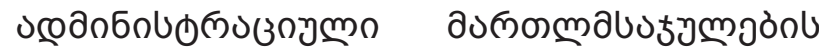

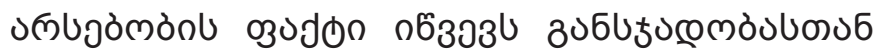

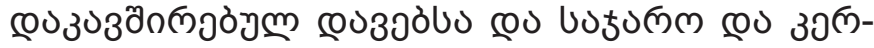

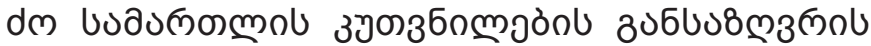

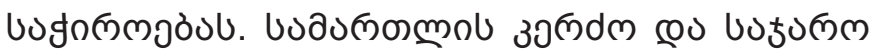

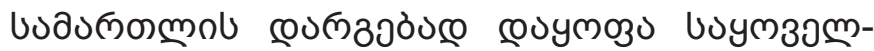

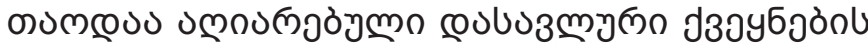

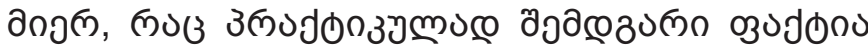

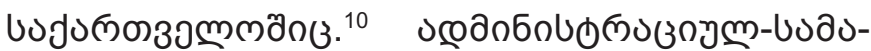

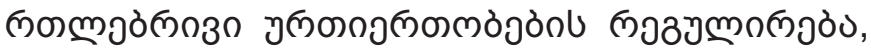

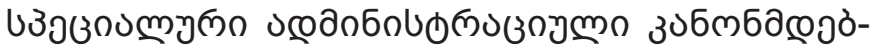

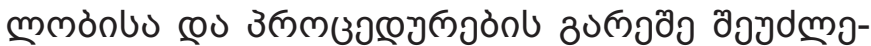

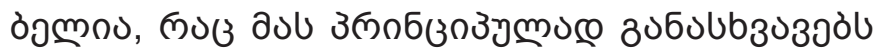

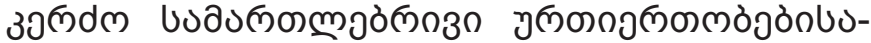

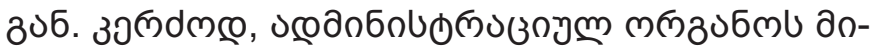

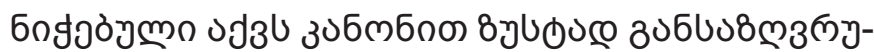

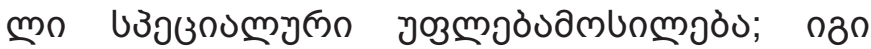

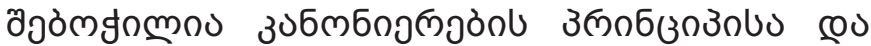

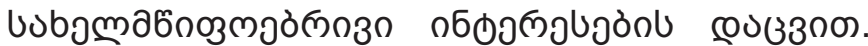

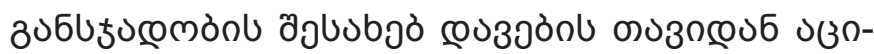

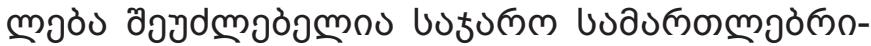

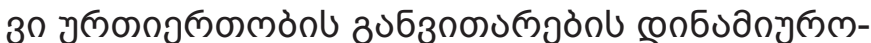

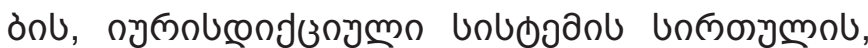

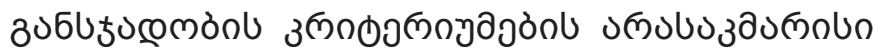

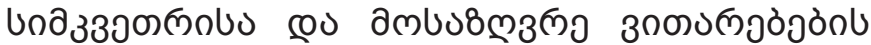

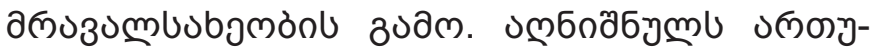

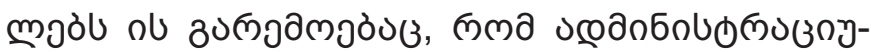

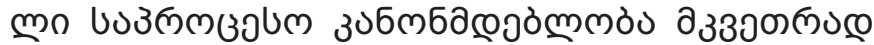
১ल аn

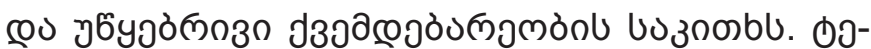

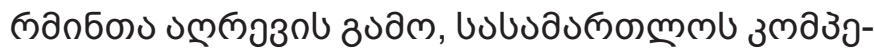

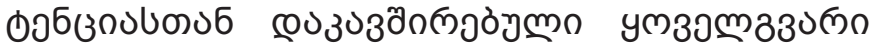

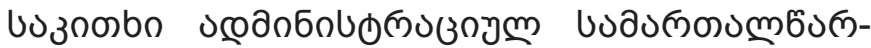

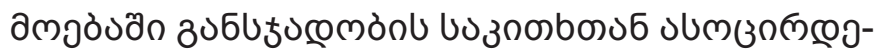

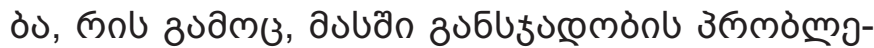

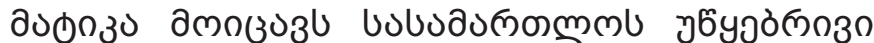

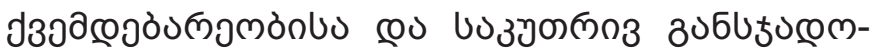

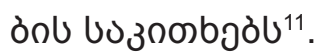

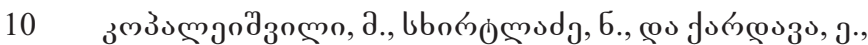

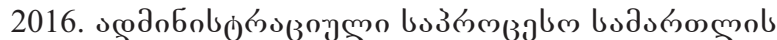

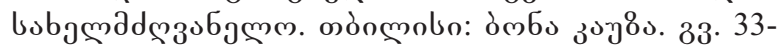
34

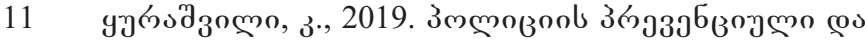

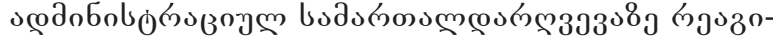

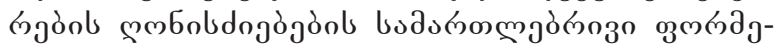

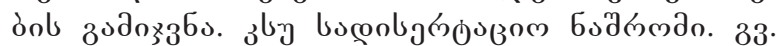
64-65. http://research.ciu.edu.ge/uploads/files/\%E1\%83 $\% 93 \%$ E $1 \% 83 \% 98 \%$ E $1 \% 83 \%$ A $1 \%$ E $1 \% 83 \% 94 \%$ E $1 \% 8$ 


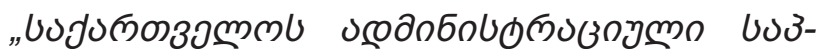

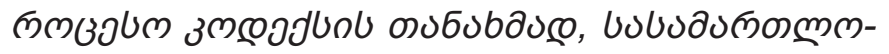

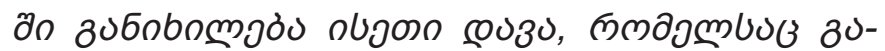

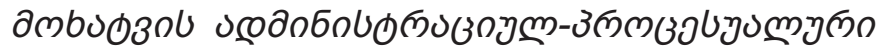

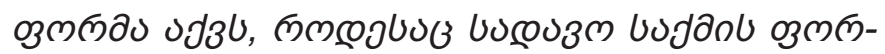

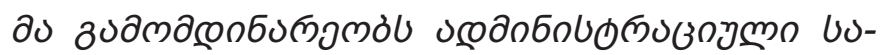

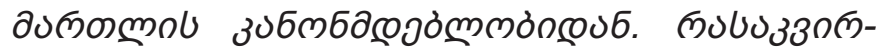

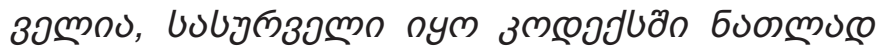

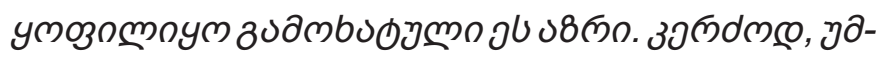

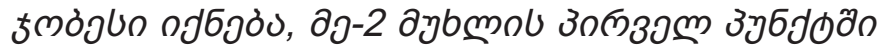

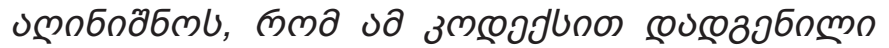

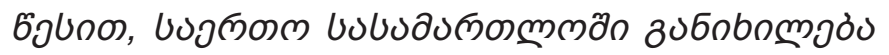

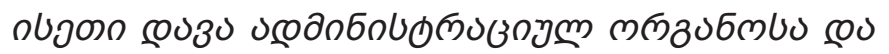

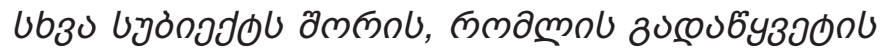

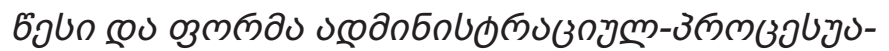

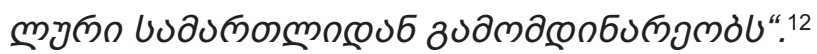

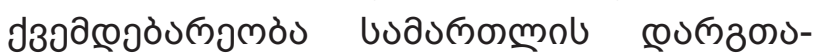

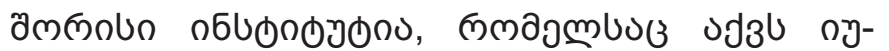

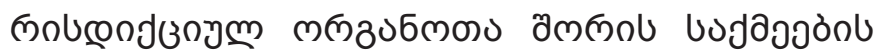

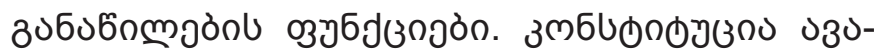

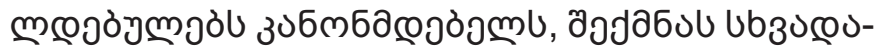

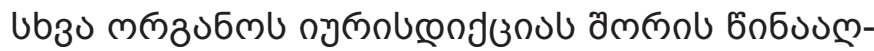

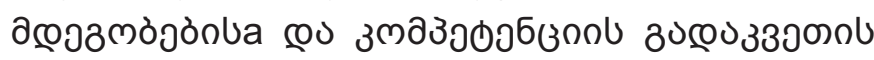

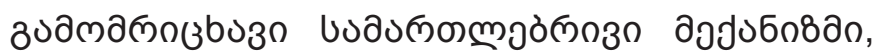

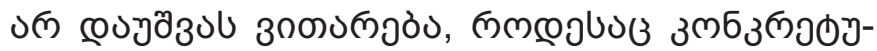

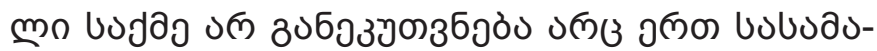

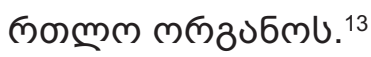

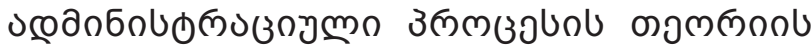

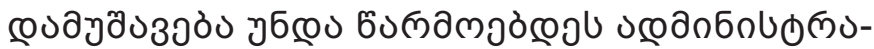

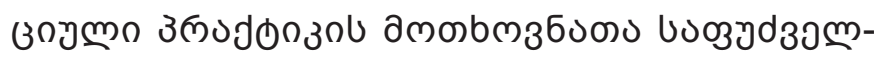

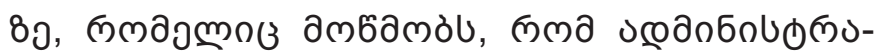

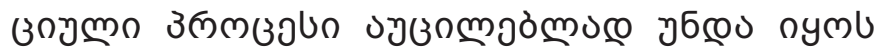

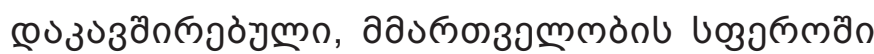

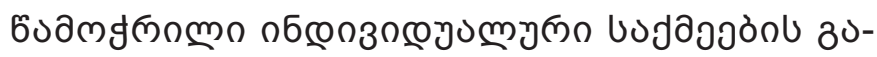
cou6y300uப⿴囗大 ${ }^{14}$.

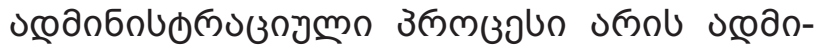

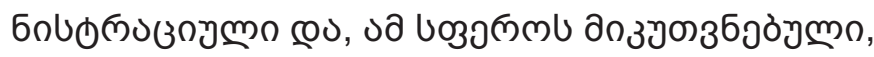

$3 \% \mathrm{~A} 0 \% \mathrm{E} 1 \% 83 \% \mathrm{~A} 2 \% \mathrm{E} 1 \% 83 \% 90 \% \mathrm{E} 1 \% 83 \% \mathrm{AA} \% \mathrm{E} 1$ $\% 83 \% 98 \%$ E $1 \% 83 \% 94 \%$ E $1 \% 83 \% 91 \%$ E $1 \% 83 \% 98 / \%$ E1 $\% 83 \% 99 \%$ E $1 \% 83 \% 90 \%$ E $1 \% 83 \%$ AE $\%$ E $\% 83 \% 90 \% 20$ $\% \mathrm{E} 1 \% 83 \% \mathrm{~A} 7 \% \mathrm{E} 1 \% 83 \% \mathrm{~A} 3 \% \mathrm{E} 1 \% 83 \% \mathrm{~A} 0 \% \mathrm{E} 1 \% 83 \% 90$ \%Е $1 \% 83 \%$ A $8 \%$ E $1 \% 83 \% 95 \%$ E $1 \% 83 \% 98 \%$ E $1 \% 83 \% 9$ A $\% \mathrm{E} 1 \% 83 \% 98 . \mathrm{pdf}$

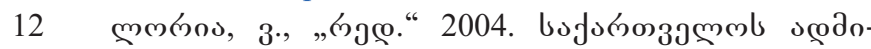

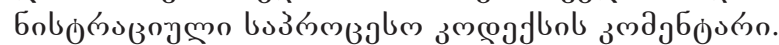

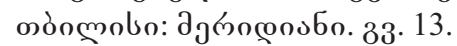

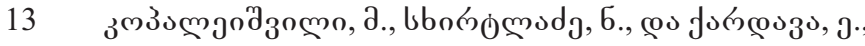

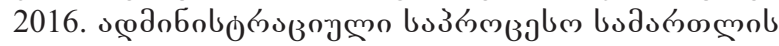

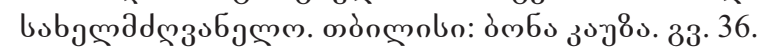

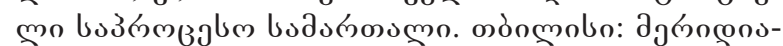
ธั. 33.27.

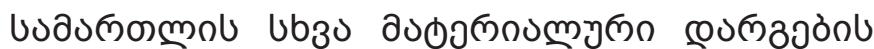

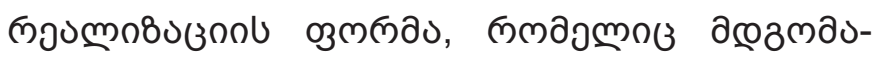

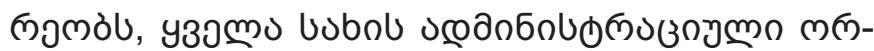

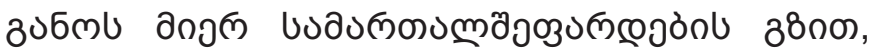

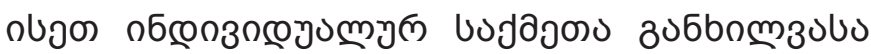

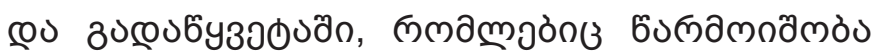

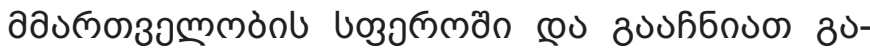

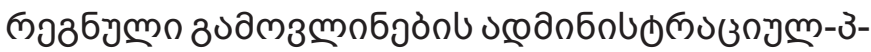

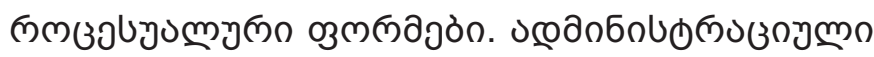

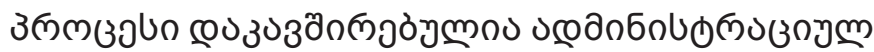

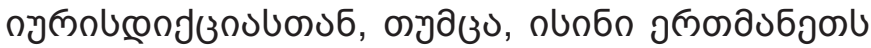

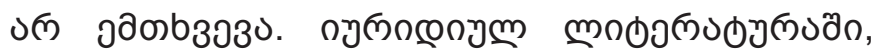

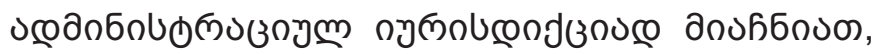

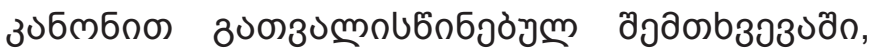

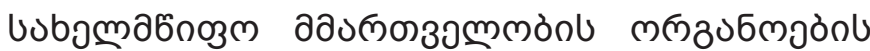

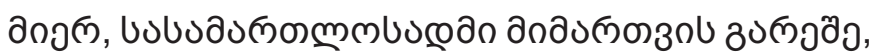

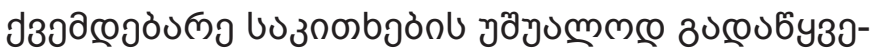

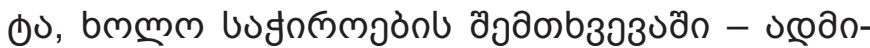

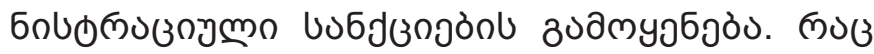

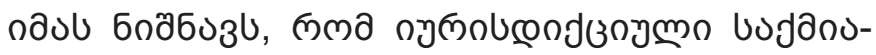

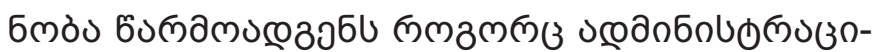

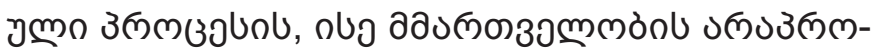

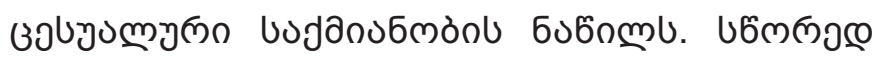

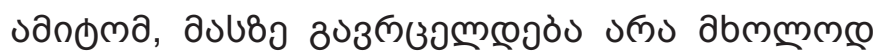

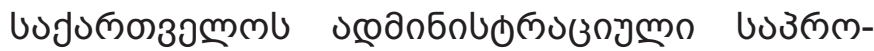

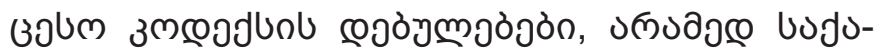

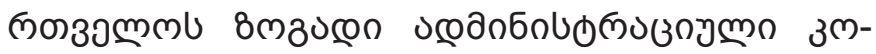

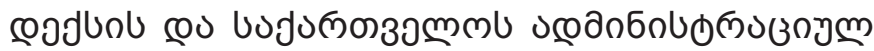

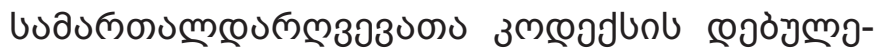

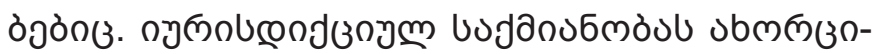

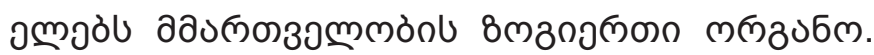

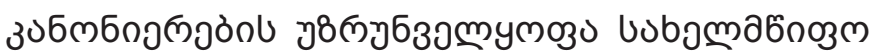

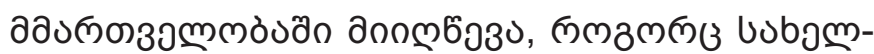

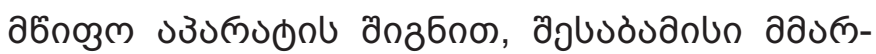

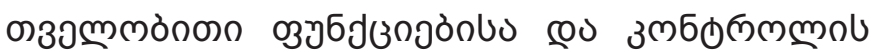

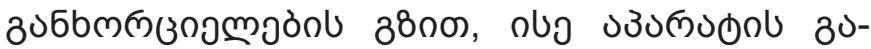

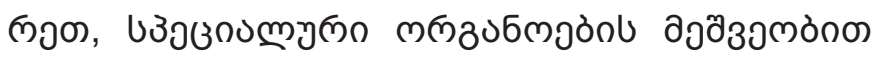

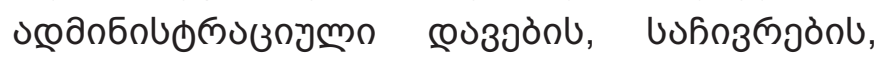

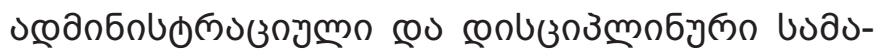

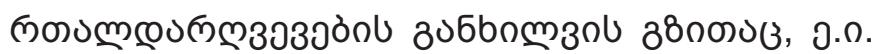

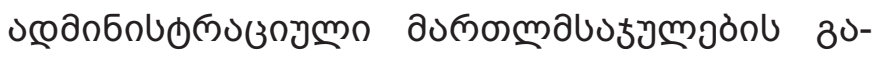

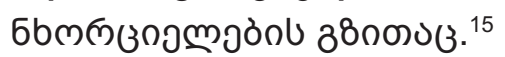

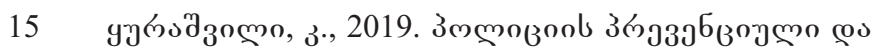

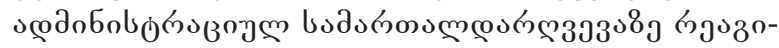

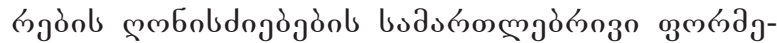

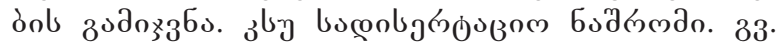
66-67. http://research.ciu.edu.ge/uploads/files/\%E1\%83 $\% 93 \%$ E $1 \% 83 \% 98 \%$ E $1 \% 83 \%$ A $1 \%$ E $1 \% 83 \% 94 \%$ E $1 \% 8$ $3 \%$ A $0 \%$ E $1 \% 83 \%$ A $2 \%$ E $1 \% 83 \% 90 \%$ E $1 \% 83 \%$ AA $\%$ E 1 \%83\%98\%E1\%83\%94\%E1\%83\%91\%E1\%83\%98/\%E1 


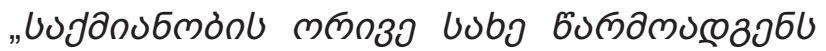
ง

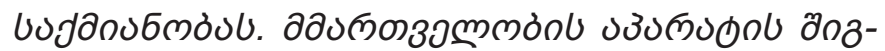

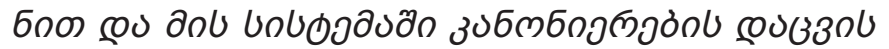

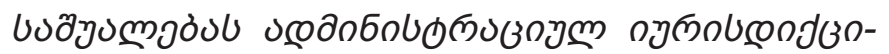

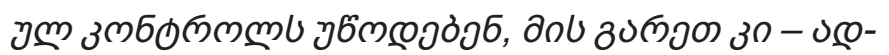

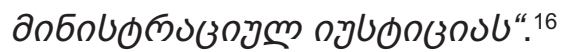

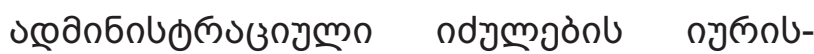

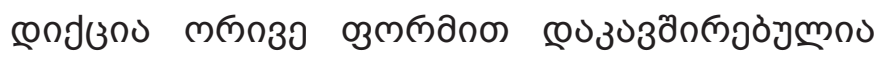

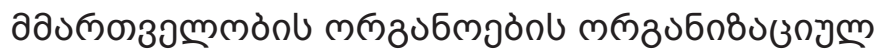

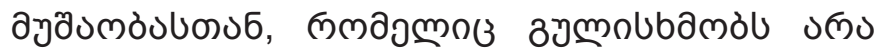

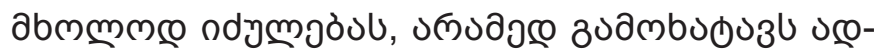

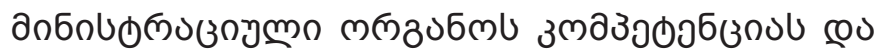

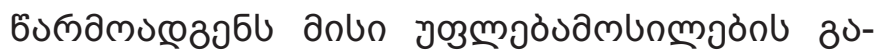

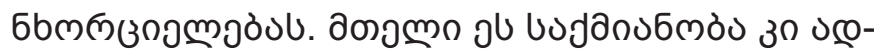

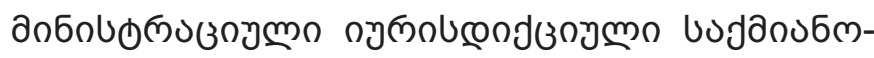

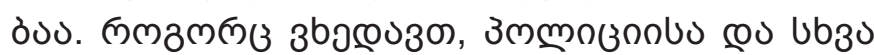

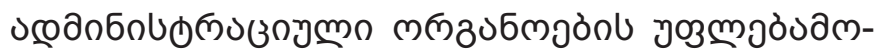

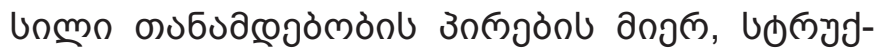

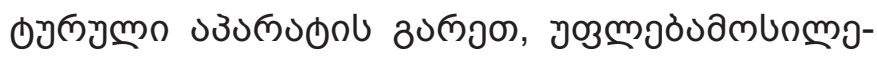

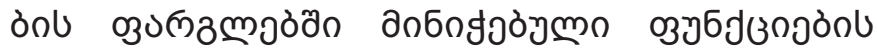

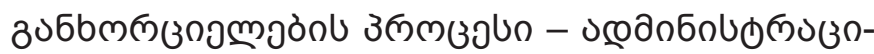

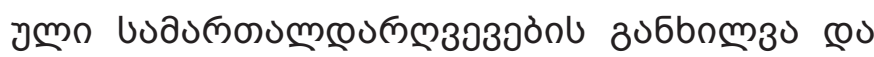

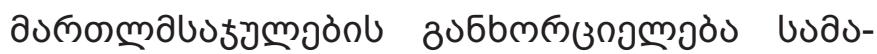

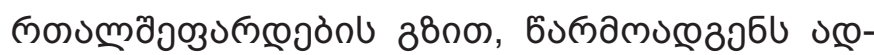

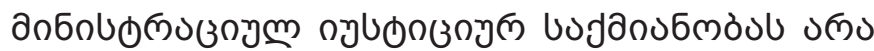

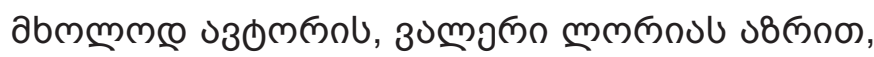

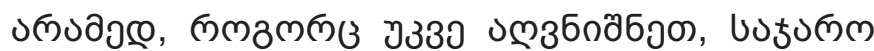

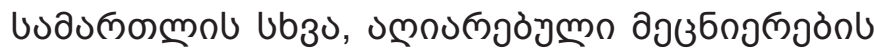

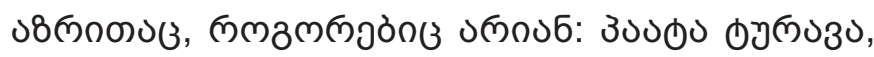

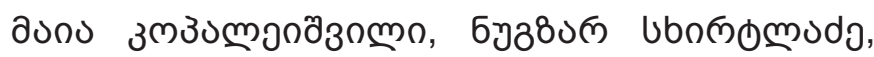

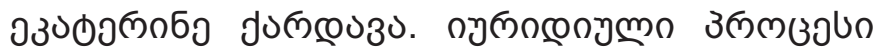

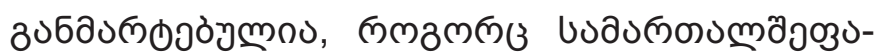

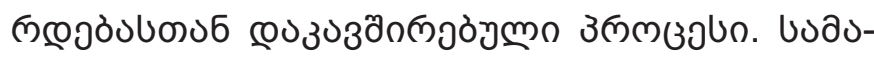

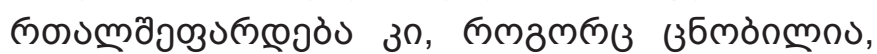

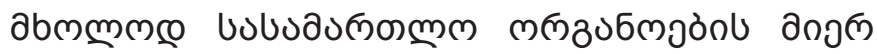

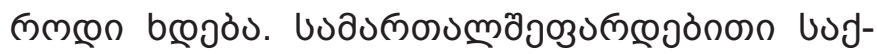

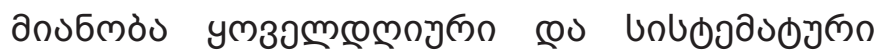

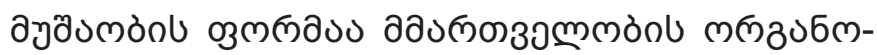

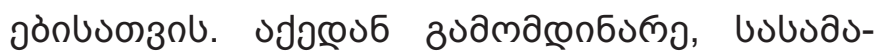

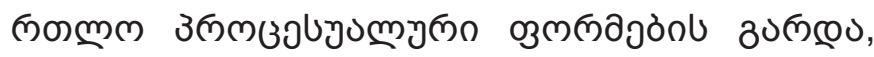

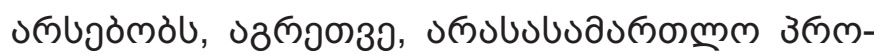

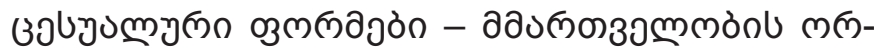

$\% 83 \% 99 \% \mathrm{E} 1 \% 83 \% 90 \% \mathrm{E} 1 \% 83 \%$ AE $\% \mathrm{E} 1 \% 83 \% 90 \% 20$ $\% \mathrm{E} 1 \% 83 \% \mathrm{~A} 7 \% \mathrm{E} 1 \% 83 \% \mathrm{~A} 3 \% \mathrm{E} 1 \% 83 \% \mathrm{~A} 0 \% \mathrm{E} 1 \% 83 \% 90$ $\% \mathrm{E} 1 \% 83 \% \mathrm{~A} 8 \% \mathrm{E} 1 \% 83 \% 95 \% \mathrm{E} 1 \% 83 \% 98 \% \mathrm{E} 1 \% 83 \% 9 \mathrm{~A}$ $\% \mathrm{E} 1 \% 83 \% 98 . \mathrm{pdf}$

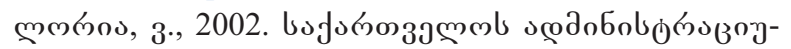

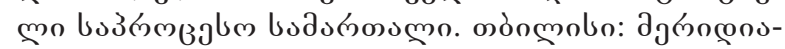
6. 33.16 .

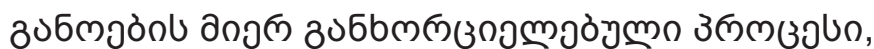

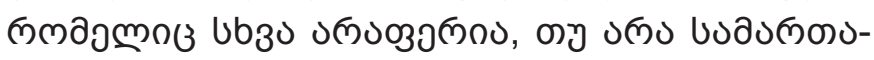

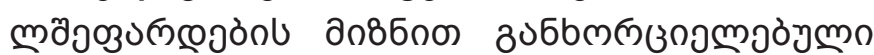

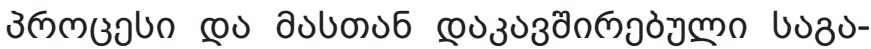

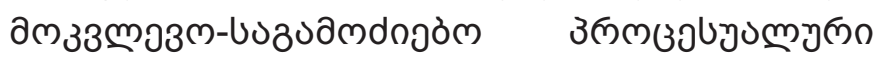

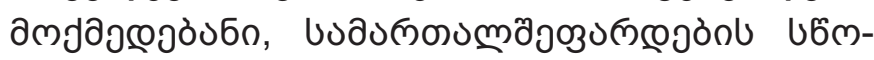

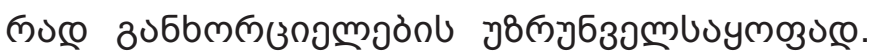

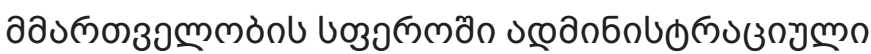

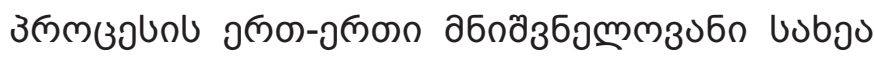

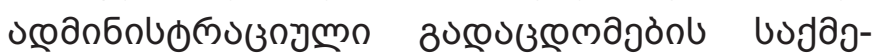

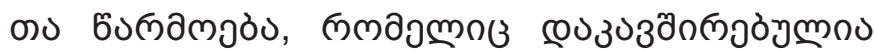

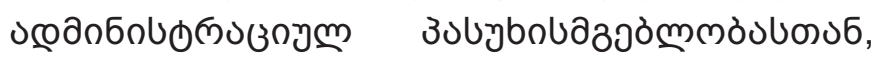

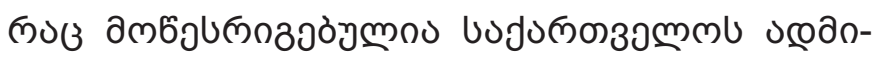

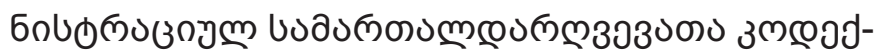

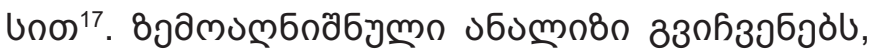

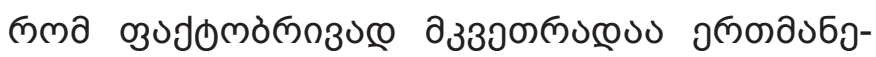

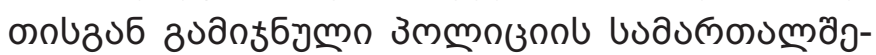

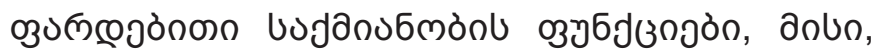

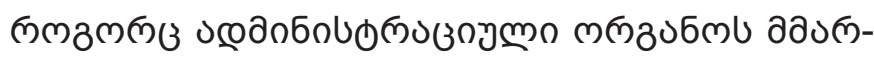

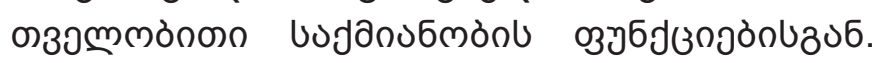

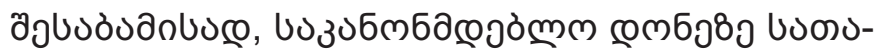

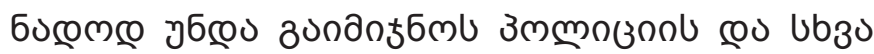

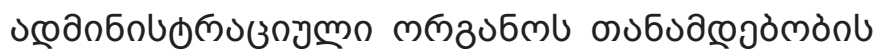

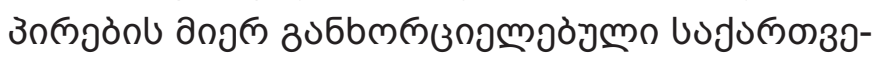

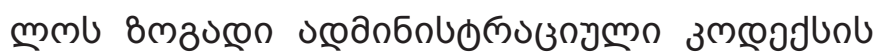

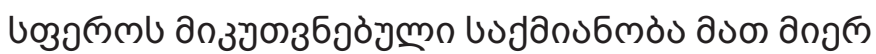

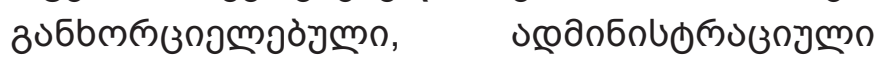

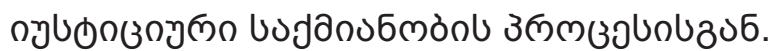

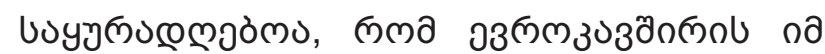

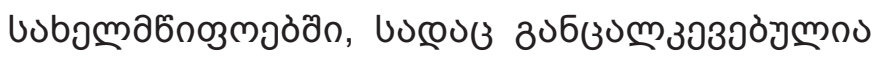

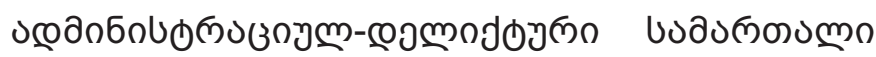

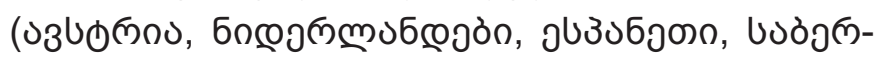

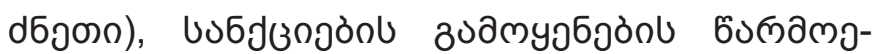

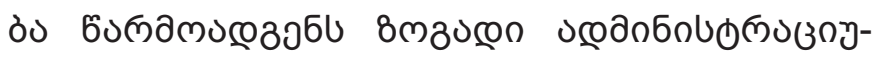

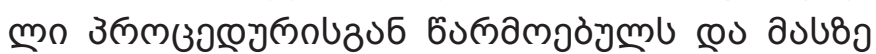

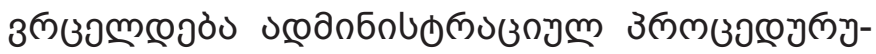

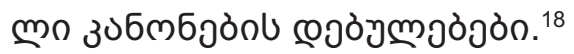

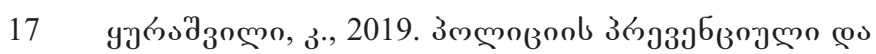

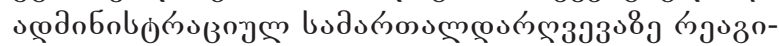

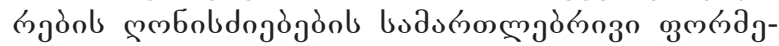

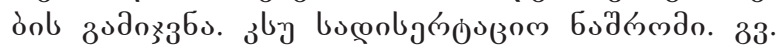
67-68. http://research.ciu.edu.ge/uploads/files/\%E1\%83 \%93\%E1\%83\%98\%E1\%83\%A1\%E1\%83\%94\%E1\%8 $3 \%$ A $0 \%$ E $1 \% 83 \%$ A $2 \%$ E $1 \% 83 \% 90 \%$ E $1 \% 83 \%$ AA $\%$ E 1 $\% 83 \% 98 \% \mathrm{E} 1 \% 83 \% 94 \% \mathrm{E} 1 \% 83 \% 91 \% \mathrm{E} 1 \% 83 \% 98 / \% \mathrm{E} 1$ $\% 83 \% 99 \%$ E $1 \% 83 \% 90 \%$ E $1 \% 83 \%$ AE $\%$ E $1 \% 83 \% 90 \% 20$ $\% \mathrm{E} 1 \% 83 \% \mathrm{~A} 7 \% \mathrm{E} 1 \% 83 \% \mathrm{~A} 3 \% \mathrm{E} 1 \% 83 \% \mathrm{~A} 0 \% \mathrm{E} 1 \% 83 \% 90$ \% $1 \% 83 \%$ A $8 \%$ E $1 \% 83 \% 95 \%$ E $1 \% 83 \% 98 \%$ E $1 \% 83 \% 9 A$ $\%$ E1\%83\%98.pdf

18 Банчук, А., 2011. Административно-деликтное законодателъство зарубежных стран. Москва: Волтерс Клувер. 33. 62. 


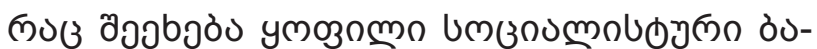

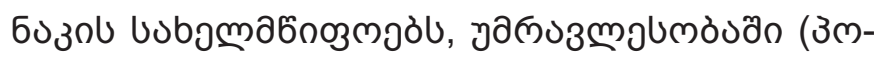

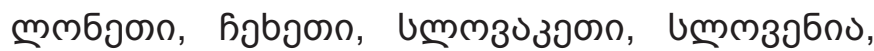

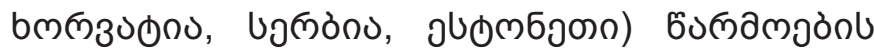

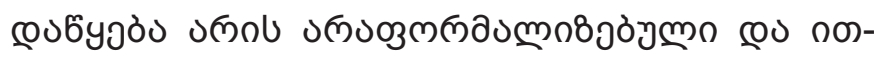

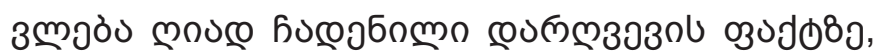

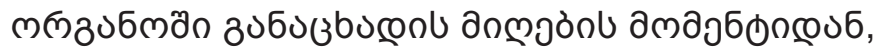

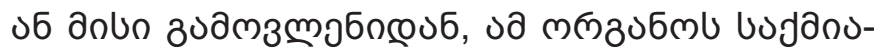

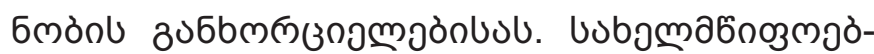

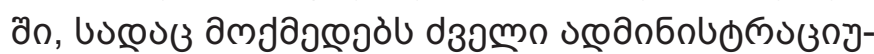

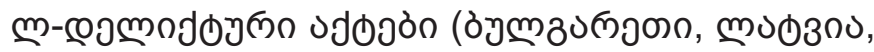

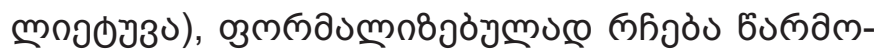

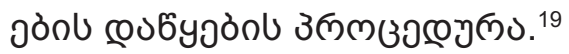

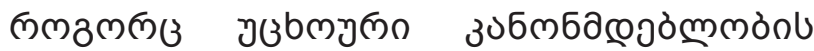

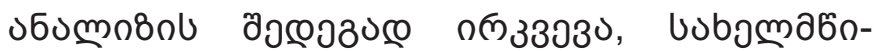

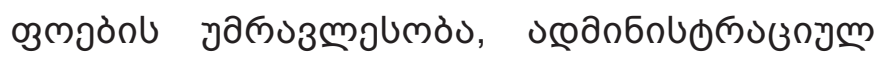

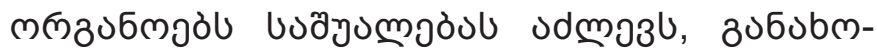

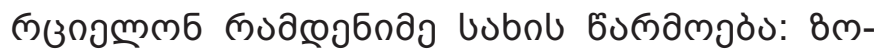

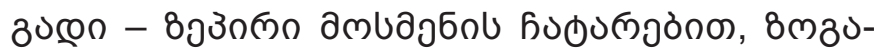

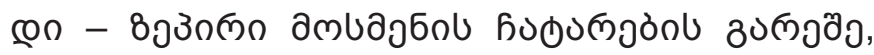

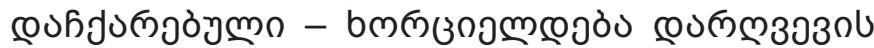

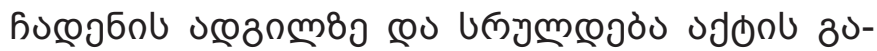

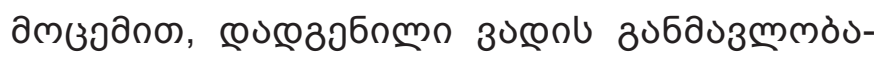

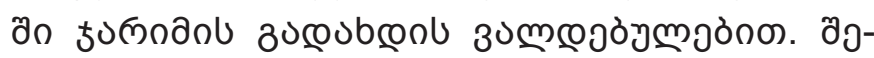

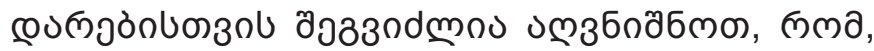

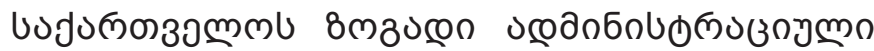

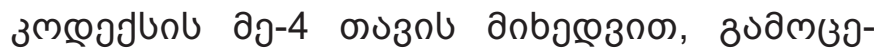

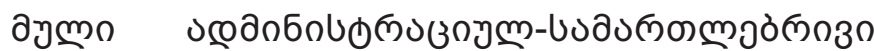

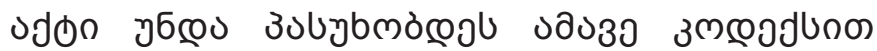

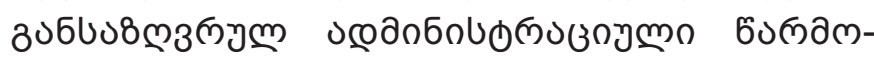

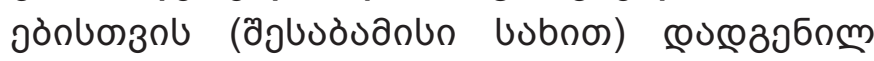

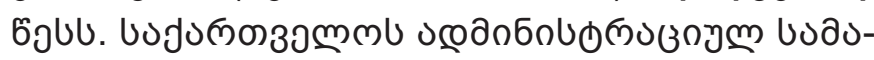

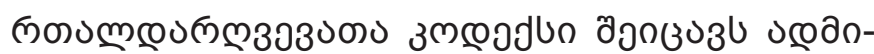

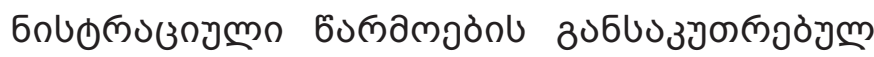

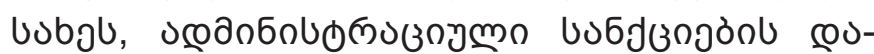

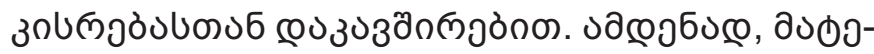

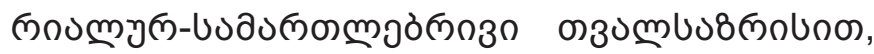

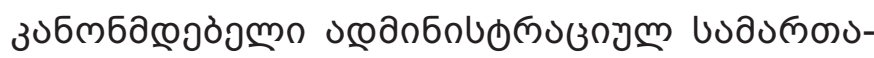

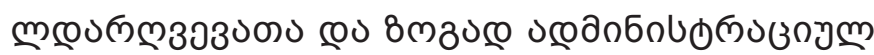

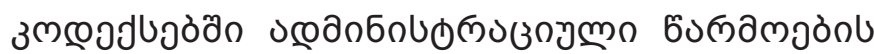

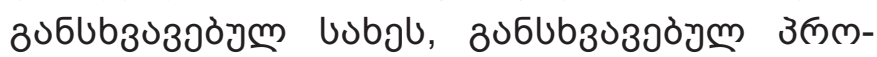

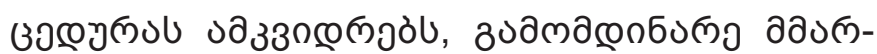

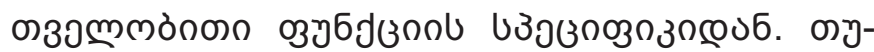

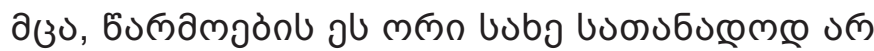

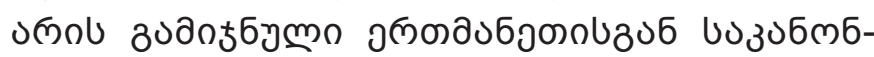

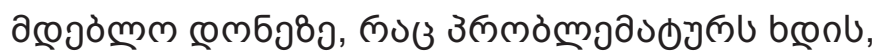

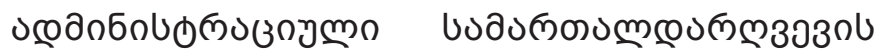

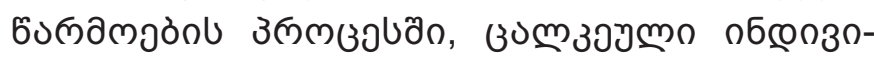

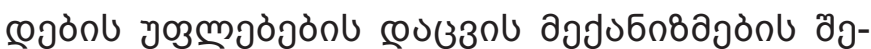
๓คววงป. ${ }^{20}$

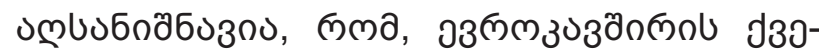

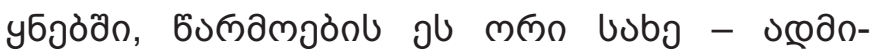

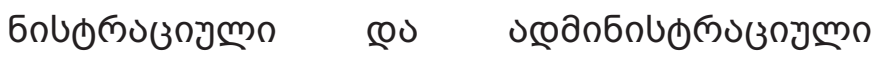

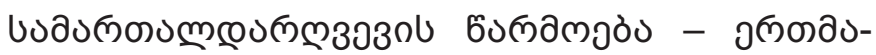

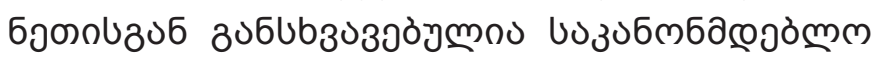

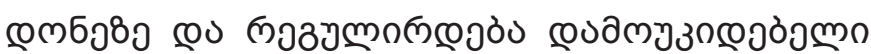

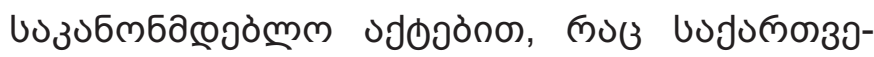

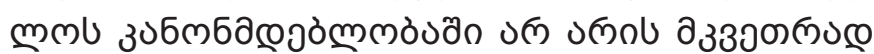

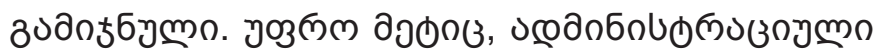

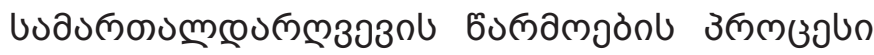

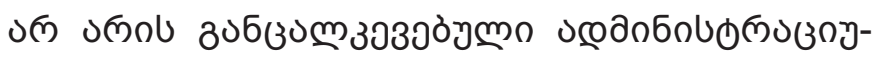

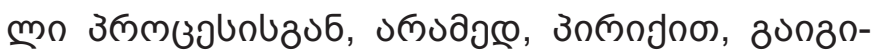

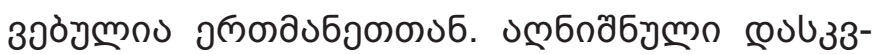

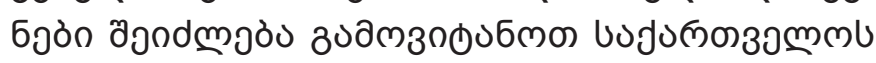

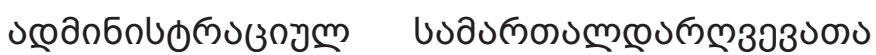

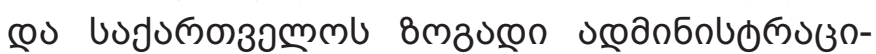

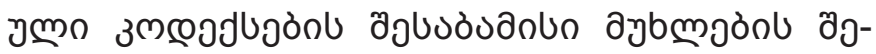

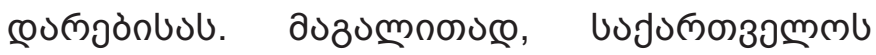

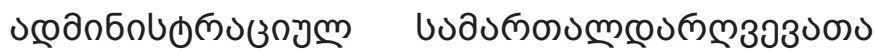

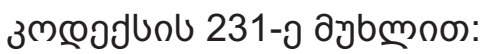

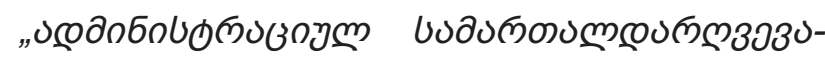

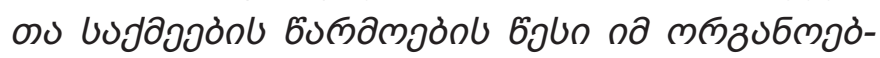

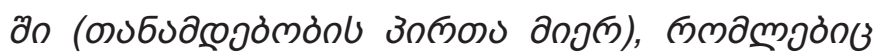

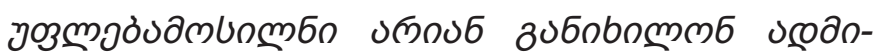

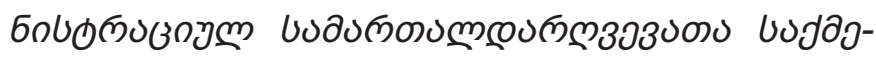

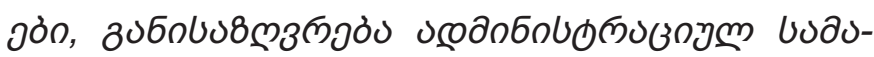

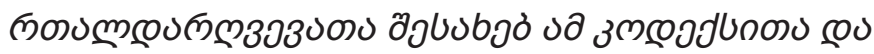

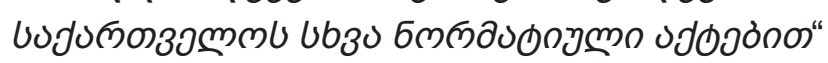

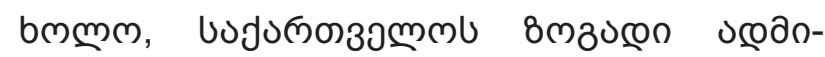

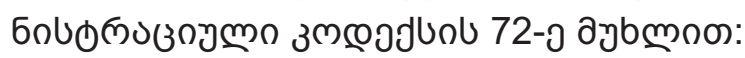

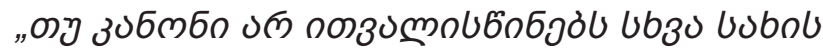
ง

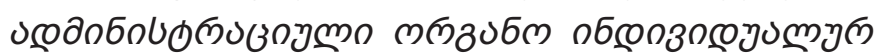

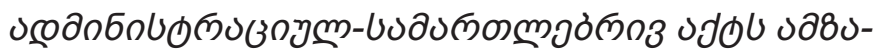

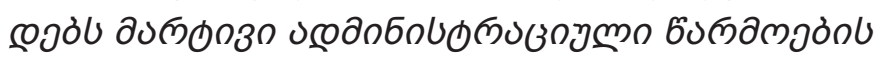
6ouno".

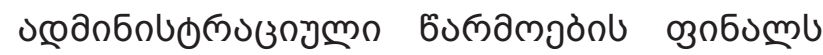

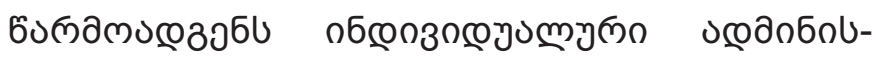

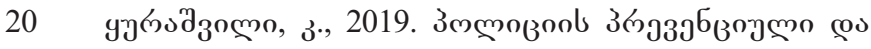

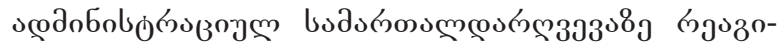

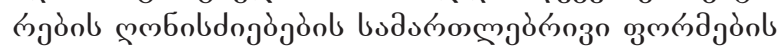

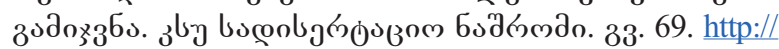
research.ciu.edu.ge/uploads/files/\%E1\%83\%93\%E1\%83\%9 8\%E1\%83\%A1\%E1\%83\%94\%E1\%83\%A0\%E1\%83\%A2 \%E1\%83\%90\%E1\%83\%AA $\%$ E1\%83\%98\%E1\%83\%94\% E1\%83\%91\%E1\%83\%98/\%E1\%83\%99\%E1\%83\%90\%E1 $\% 83 \%$ AE $\%$ E $1 \% 83 \% 90 \% 20 \%$ E $1 \% 83 \%$ A $7 \%$ E $1 \% 83 \%$ A $3 \%$ E1\%83\%A0\%E1\%83\%90\%E1\%83\%A8\%E1\%83\%95\%E1 \%83\%98\%E1\%83\%9A\%E1\%83\%98.pdf 


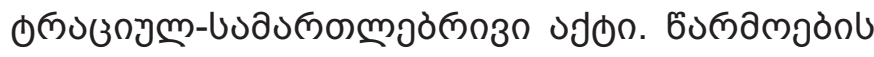

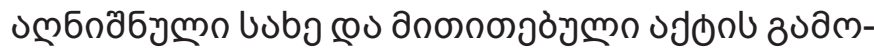

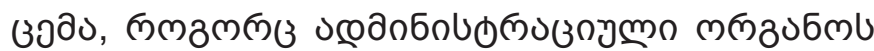

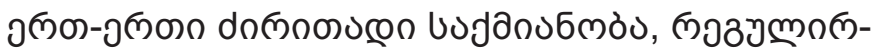

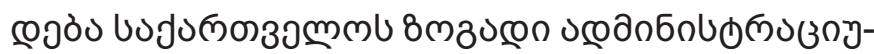

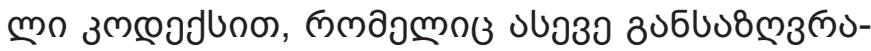

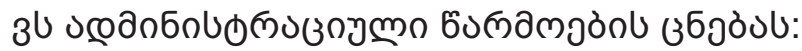

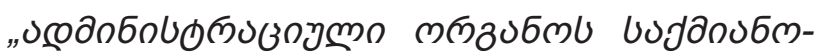

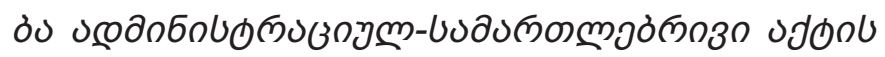

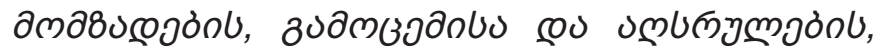

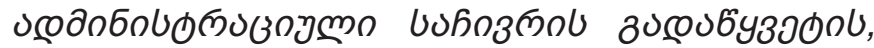

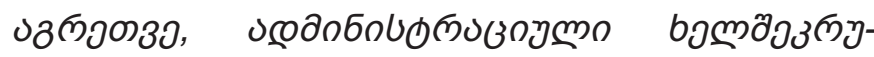

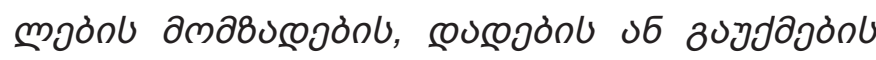

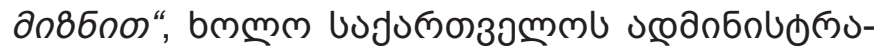

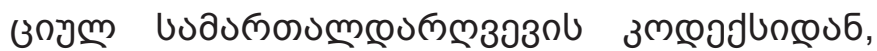

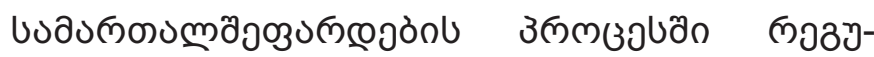

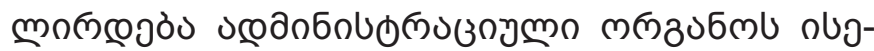

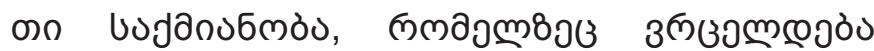

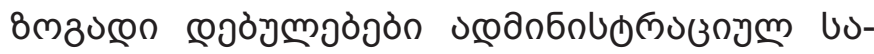

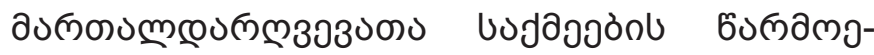

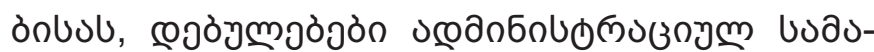

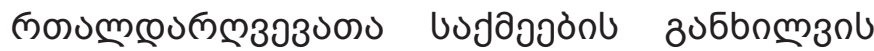

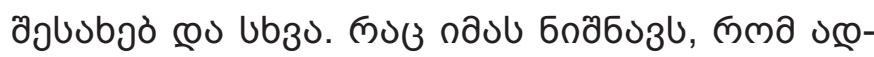

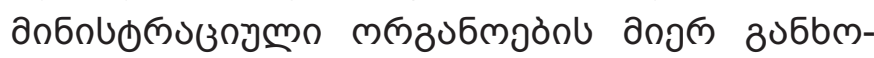

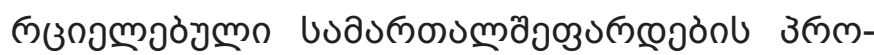

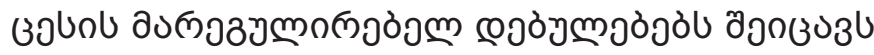

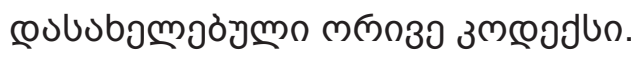

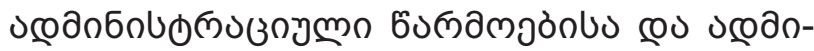

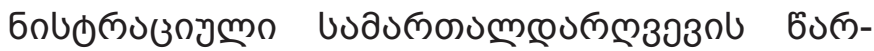

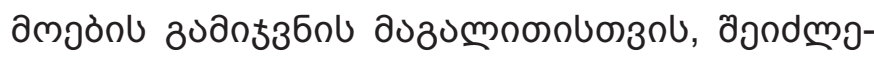

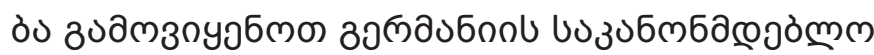

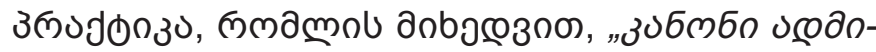

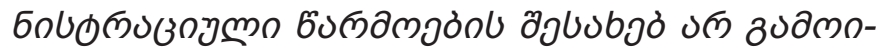

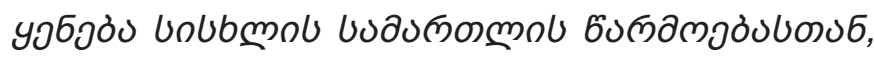
ง

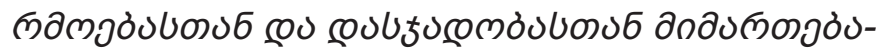

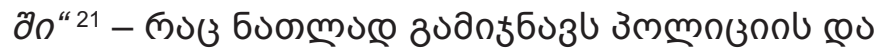

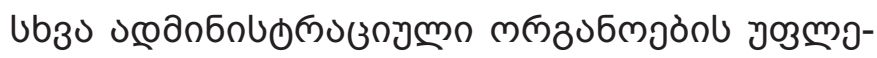

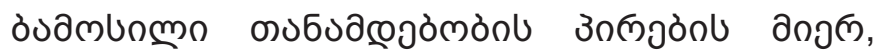

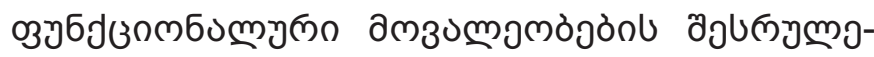

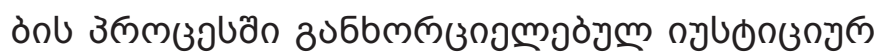

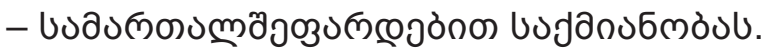

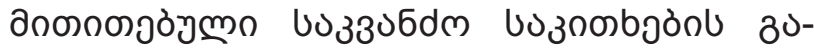

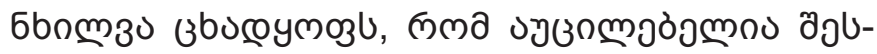

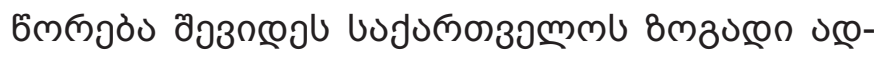

21 Бергманн, В., 2013. Административно-процессуальное право Германии, Закон об Административном производстве Германии. (2-ое издание). Москва-Берлин: «infotropic media». 33.9 .

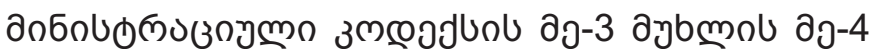

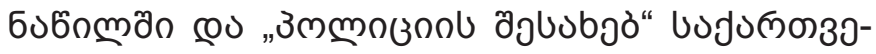

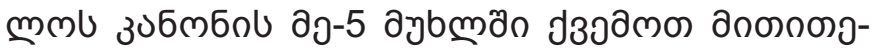

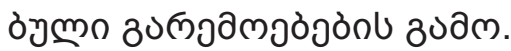

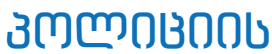

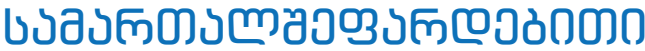

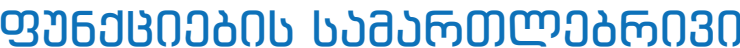

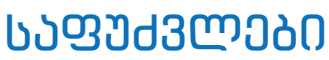

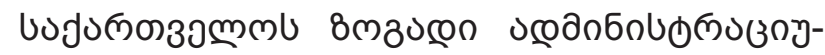

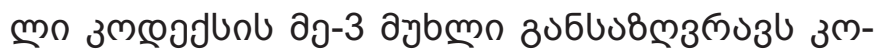

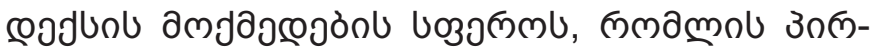

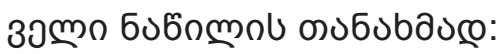

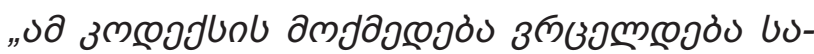

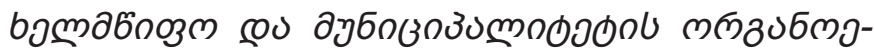
onbu ço ç

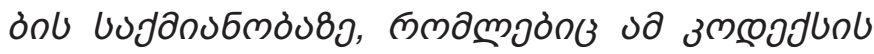

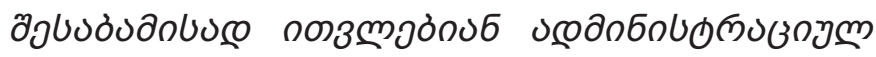

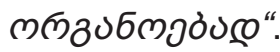

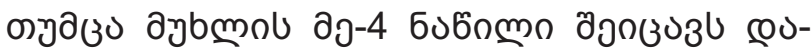

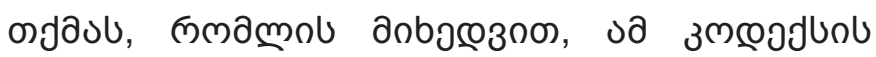

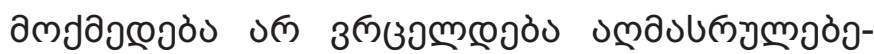

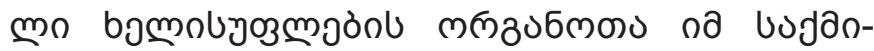

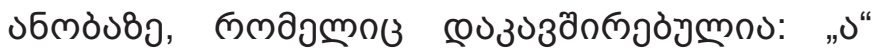

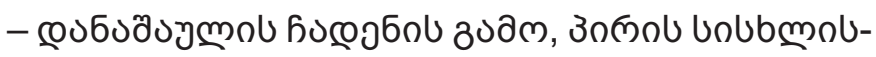

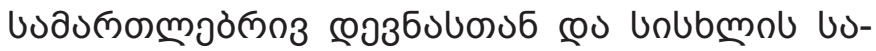

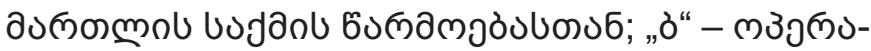

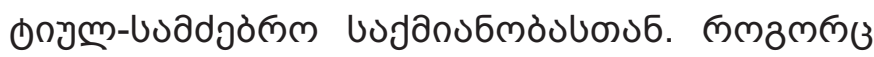

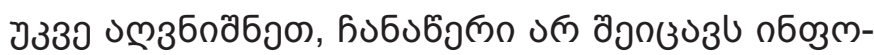

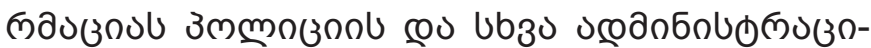

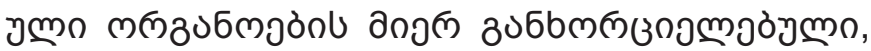

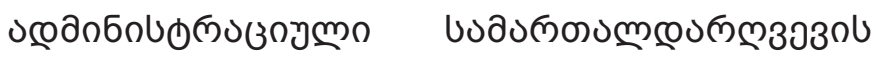

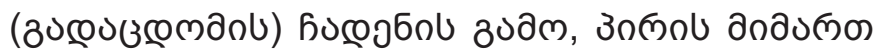

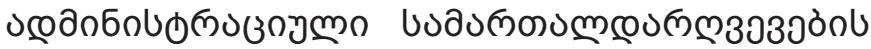

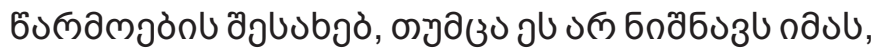

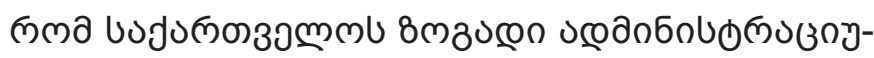

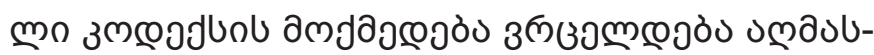

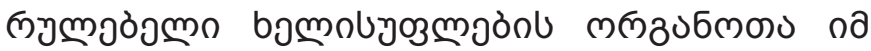

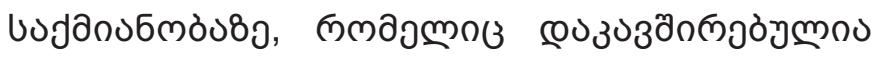

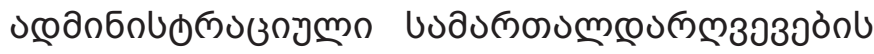

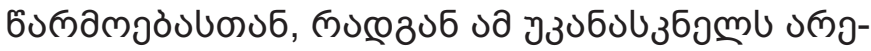

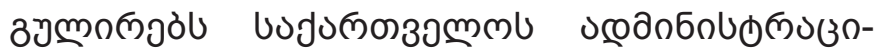

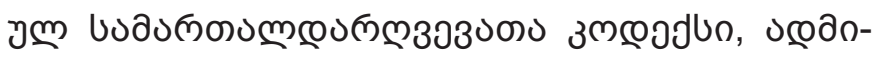

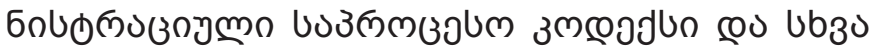

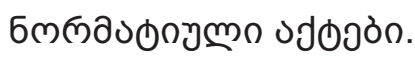

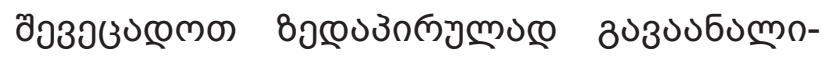

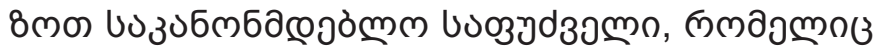




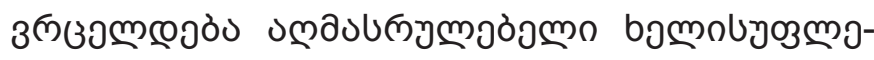

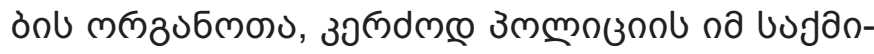

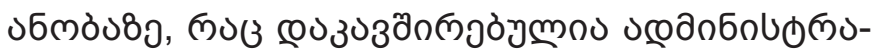

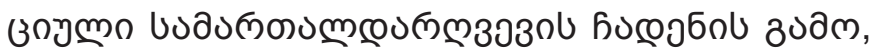

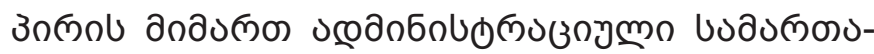

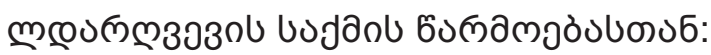

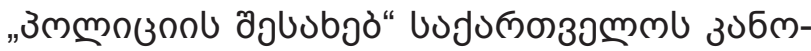

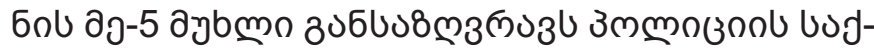

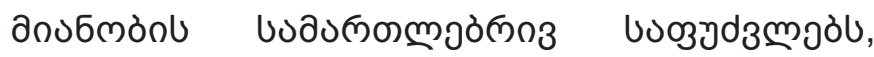

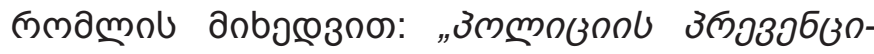

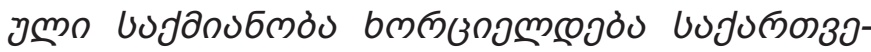

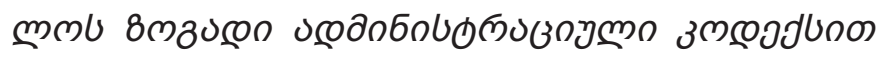

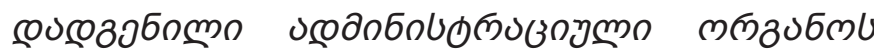

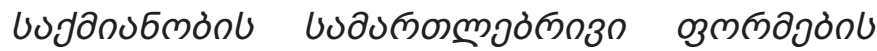

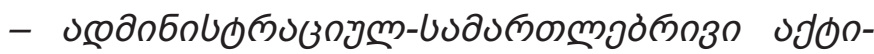

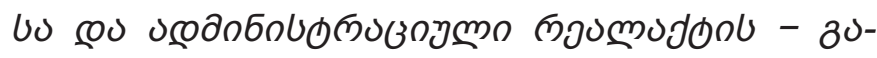

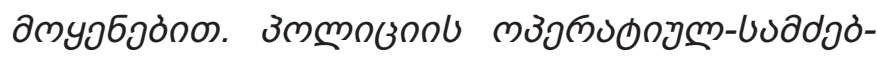

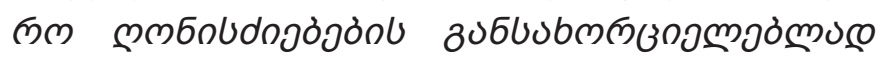

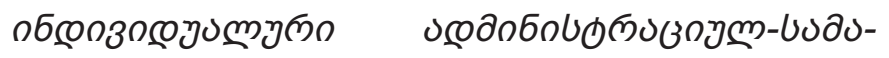

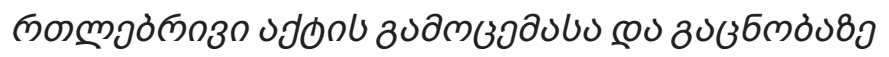

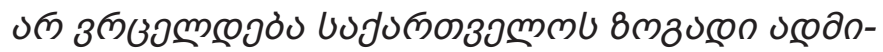

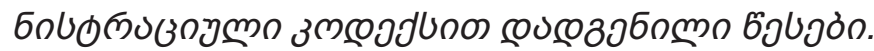

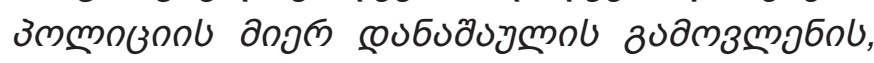

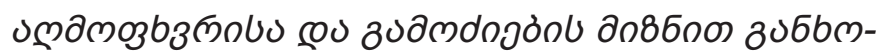

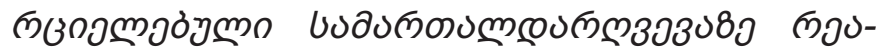

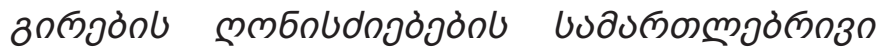

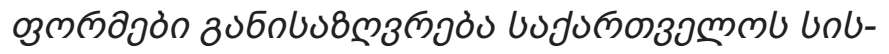

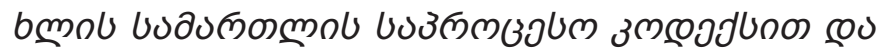

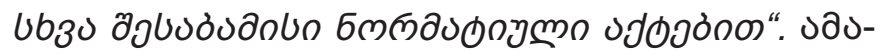

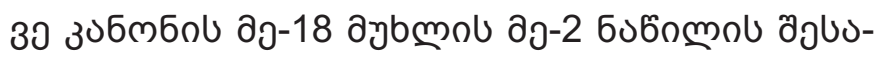

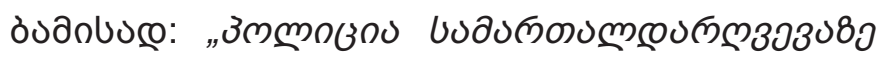

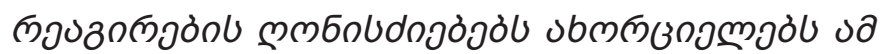

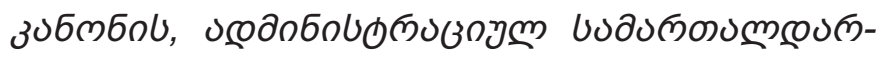

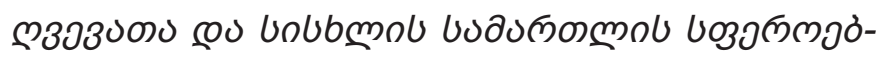

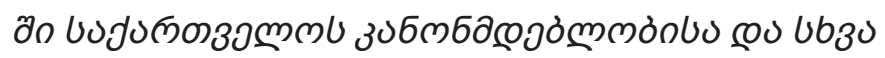

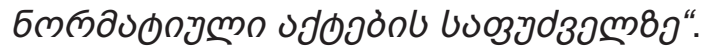

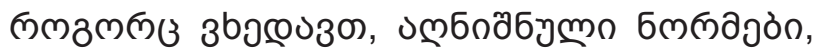

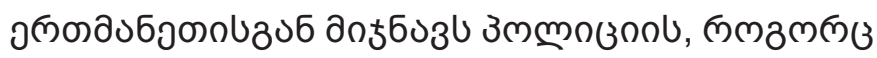

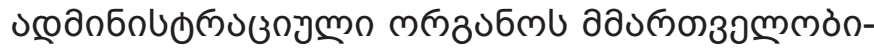

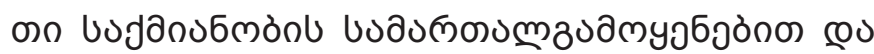

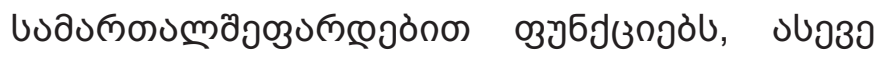

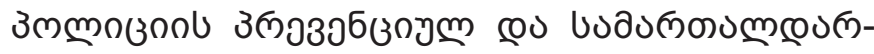

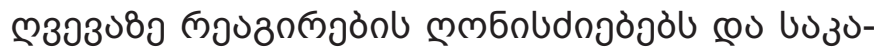

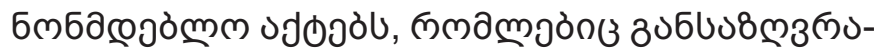

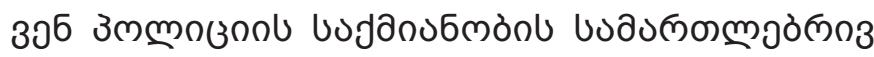

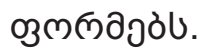

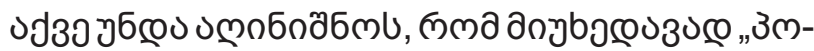

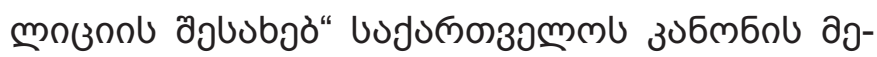

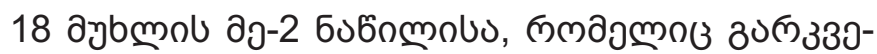

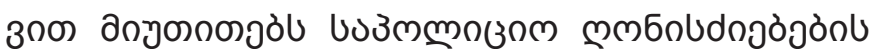

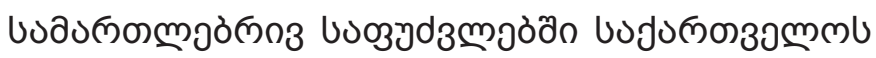

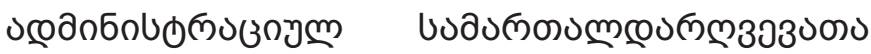

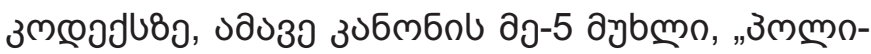

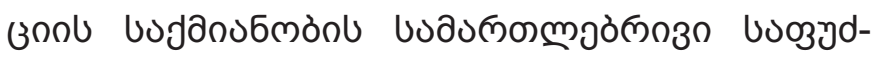

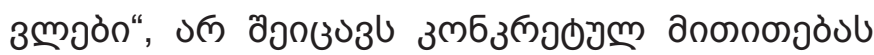

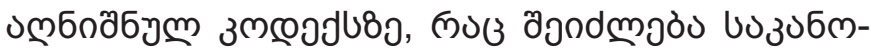

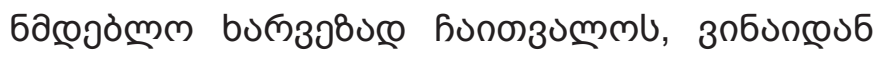

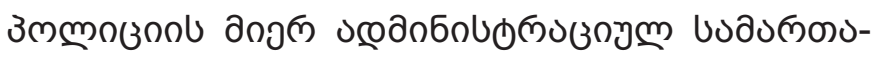

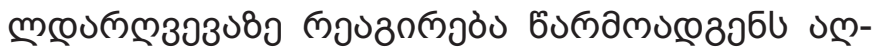

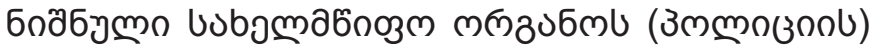

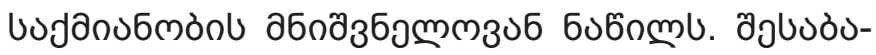

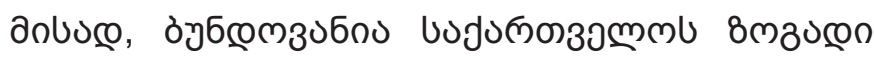

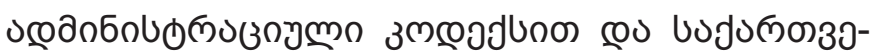

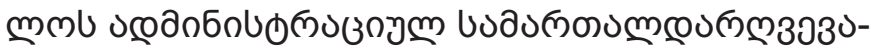

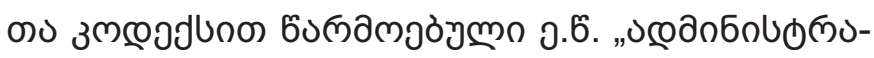

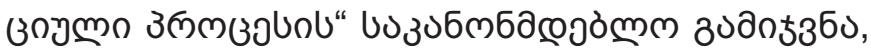

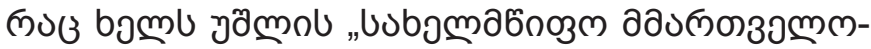

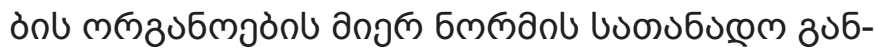

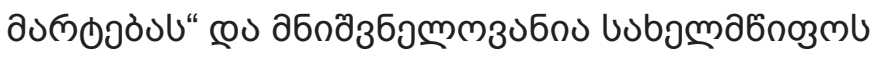

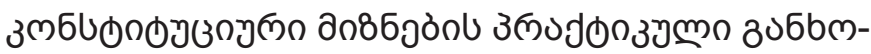

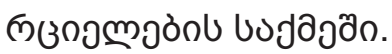

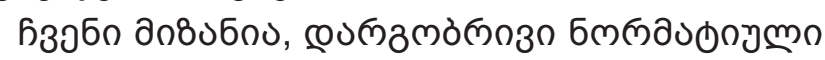

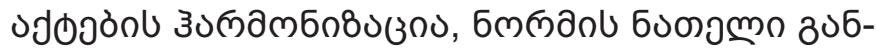

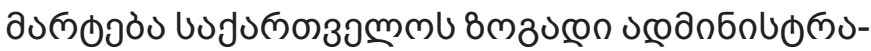

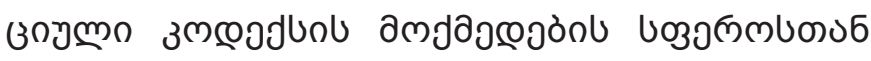

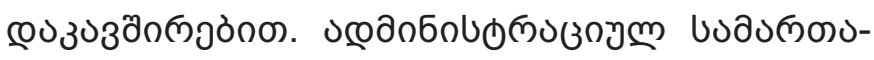

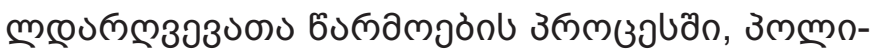

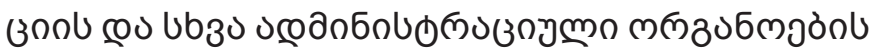

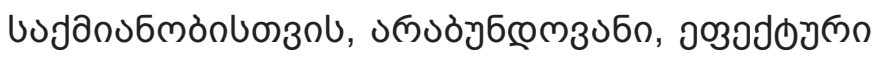

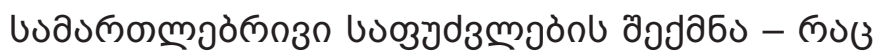

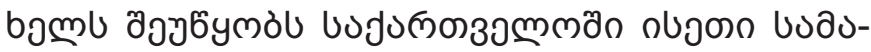

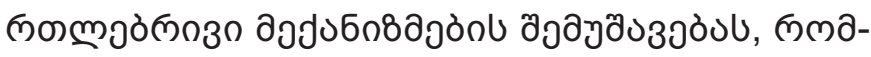

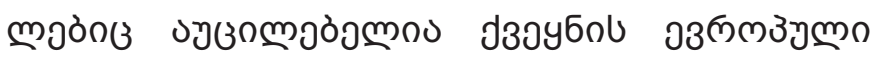

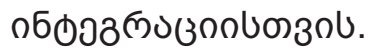

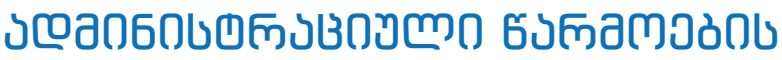

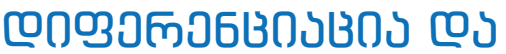

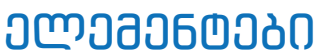

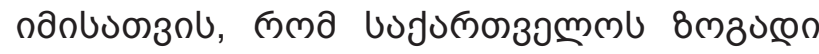

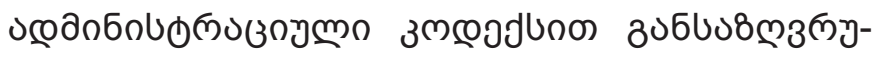

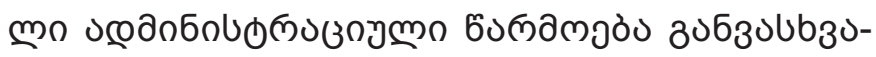

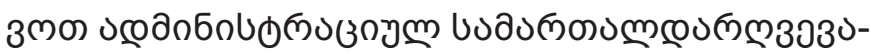

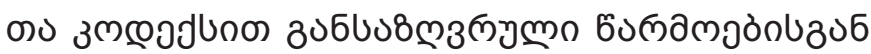

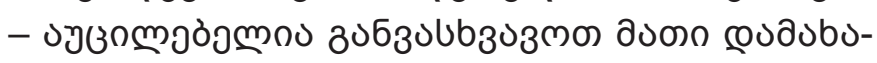

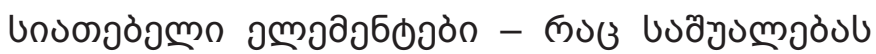




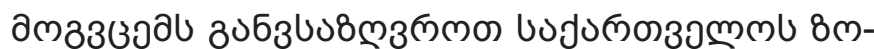

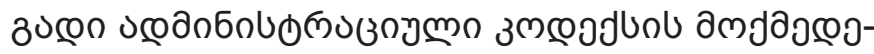
onb பоुगलм:

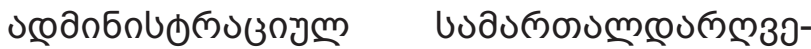

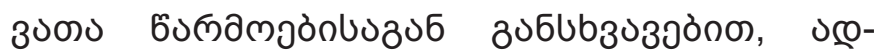

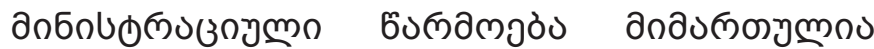

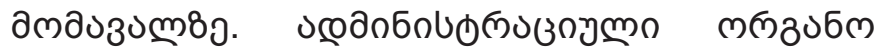

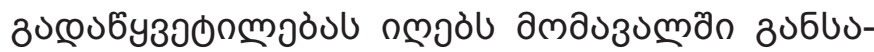

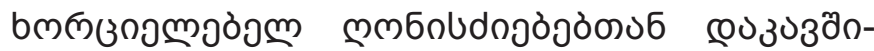

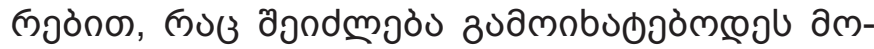

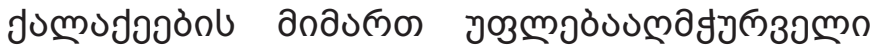

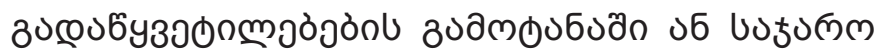

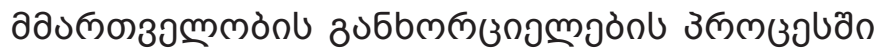

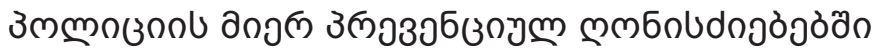

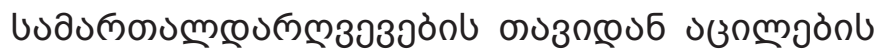

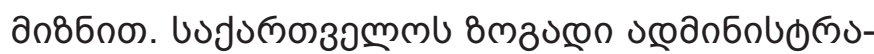

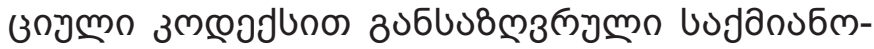

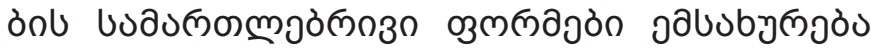

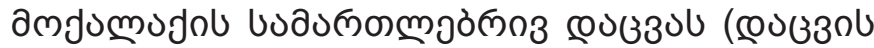

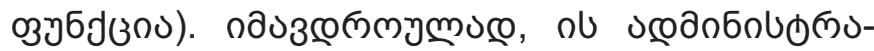

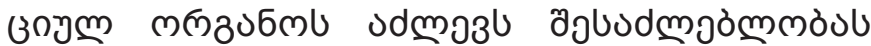

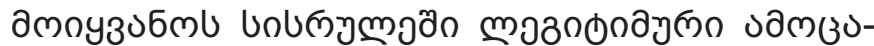

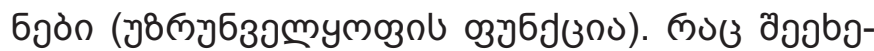

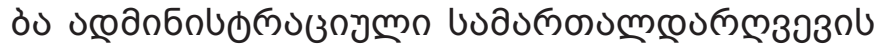

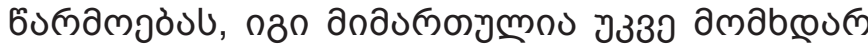

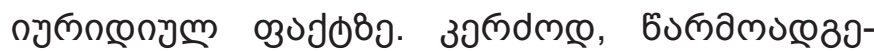

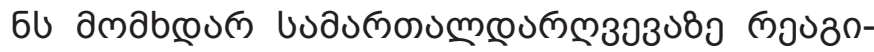

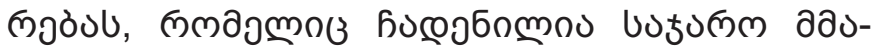

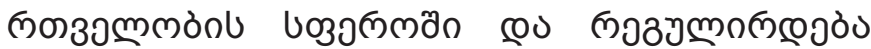

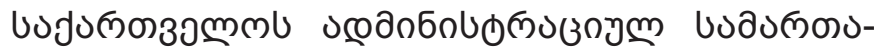

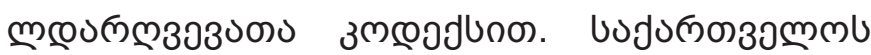
ง

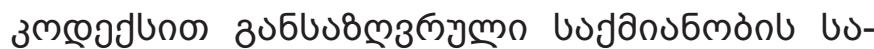

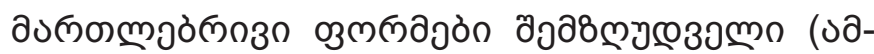

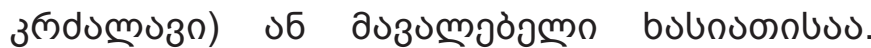

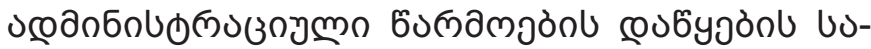

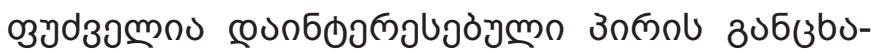

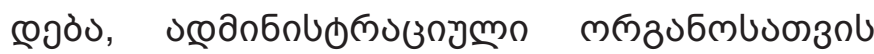

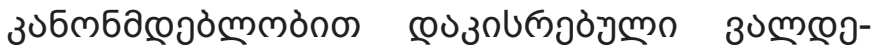

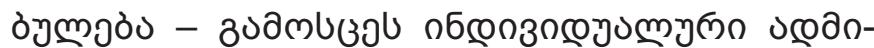

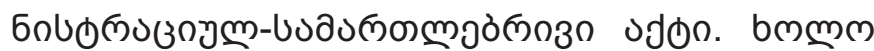

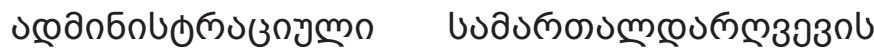

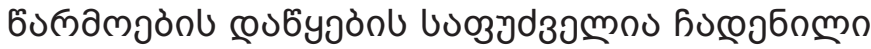

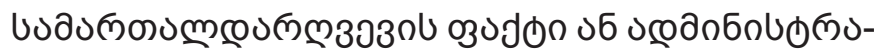

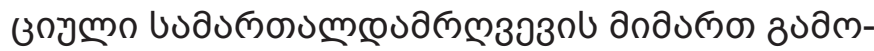

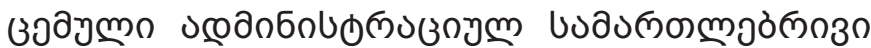

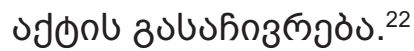

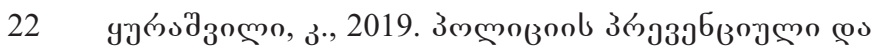

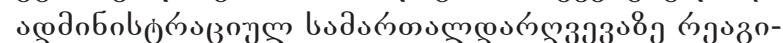

ง

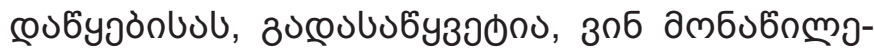

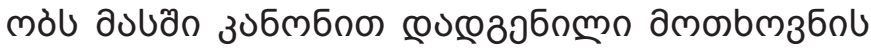

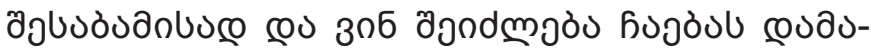

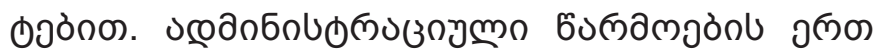

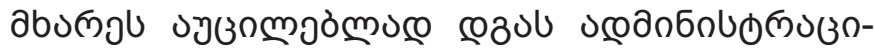

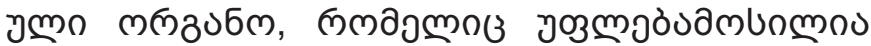

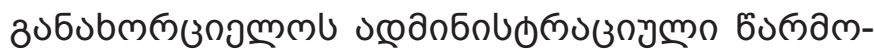
јอง

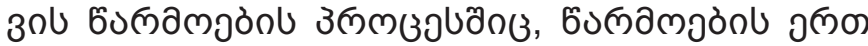

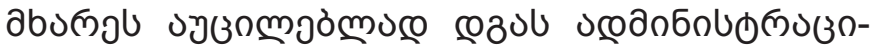

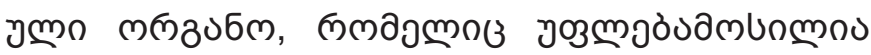

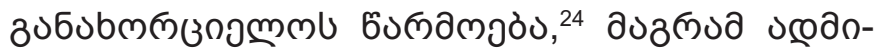

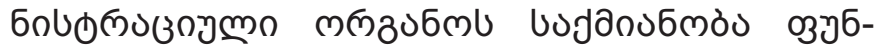

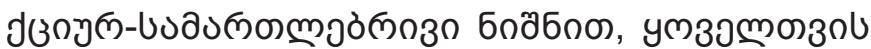

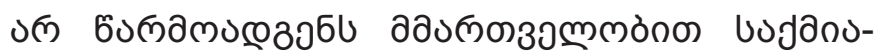

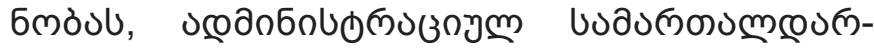

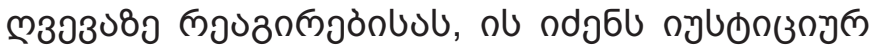

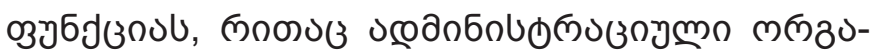

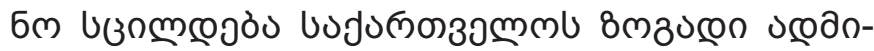

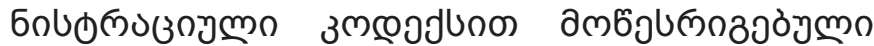

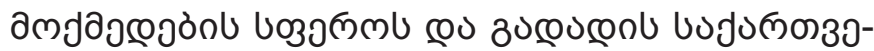

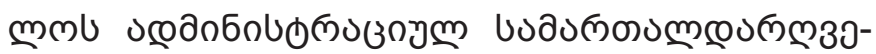

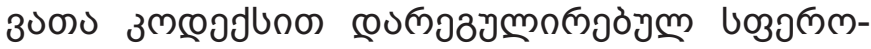

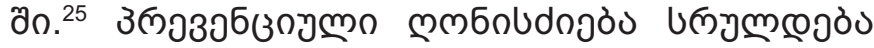

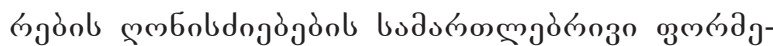
òn зодоз 74-75. http://research.ciu.edu.ge/uploads/files/\%E1\%83 $\% 93 \%$ E $1 \% 83 \% 98 \%$ E $1 \% 83 \%$ A1\%E1\%83\%94\%E1\%8 $3 \%$ A $0 \%$ E $1 \% 83 \%$ A $2 \%$ E $1 \% 83 \% 90 \%$ E $1 \% 83 \%$ AA $\%$ E 1 $\% 83 \% 98 \% \mathrm{E} 1 \% 83 \% 94 \% \mathrm{E} 1 \% 83 \% 91 \% \mathrm{E} 1 \% 83 \% 98 / \% \mathrm{E} 1$ $\% 83 \% 99 \%$ E $1 \% 83 \% 90 \%$ E $1 \% 83 \%$ AE $\%$ E $\% 83 \% 90 \% 20$ $\% \mathrm{E} 1 \% 83 \% \mathrm{~A} 7 \% \mathrm{E} 1 \% 83 \% \mathrm{~A} 3 \% \mathrm{E} 1 \% 83 \% \mathrm{~A} 0 \% \mathrm{E} 1 \% 83 \% 90$ $\%$ E $1 \% 83 \%$ A $8 \%$ E $1 \% 83 \% 95 \%$ E $1 \% 83 \% 98 \%$ E $1 \% 83 \% 9 A$ $\% \mathrm{E} 1 \% 83 \% 98 . \mathrm{pdf}$

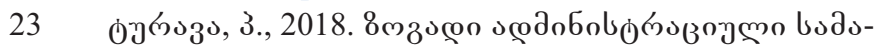

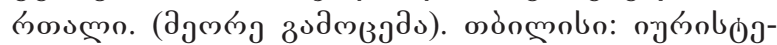
òbl bodyornm. 33.222

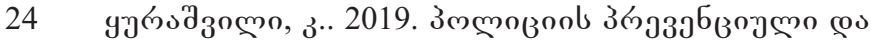

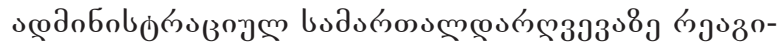

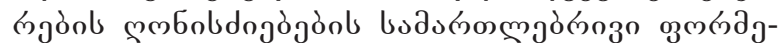

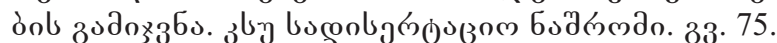
http://research.ciu.edu.ge/uploads/files/\%E1\%83\%93\%E $1 \% 83 \% 98 \% \mathrm{E} 1 \% 83 \% \mathrm{~A} 1 \% \mathrm{E} 1 \% 83 \% 94 \% \mathrm{E} 1 \% 83 \% \mathrm{~A} 0 \% \mathrm{E}$ 1\%83\%A2\%E1\%83\%90\%E1\%83\%AA \%E1\%83\%98\%E $1 \% 83 \% 94 \% \mathrm{E} 1 \% 83 \% 91 \% \mathrm{E} 1 \% 83 \% 98 / \% \mathrm{E} 1 \% 83 \% 99 \% \mathrm{E} 1$ $\% 83 \% 90 \%$ E $1 \% 83 \%$ AE $\%$ E $1 \% 83 \% 90 \% 20 \%$ E $1 \% 83 \%$ A7 $\% \mathrm{E} 1 \% 83 \% \mathrm{~A} 3 \% \mathrm{E} 1 \% 83 \% \mathrm{~A} 0 \% \mathrm{E} 1 \% 83 \% 90 \% \mathrm{E} 1 \% 83 \% \mathrm{~A} 8$ \%E1\%83\%95\%E1\%83\%98\%E1\%83\%9A\%E1\%83\%98. pdf

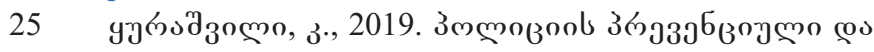

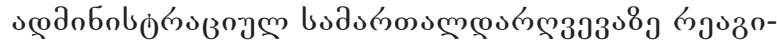

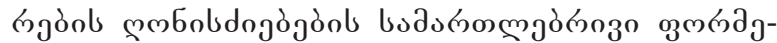

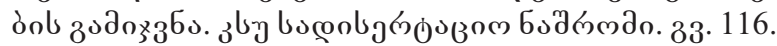
http://research.ciu.edu.ge/uploads/files/\%E1\%83\%93\%E 


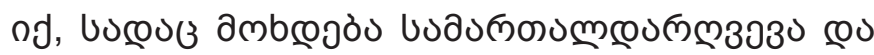

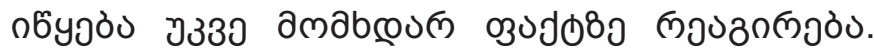

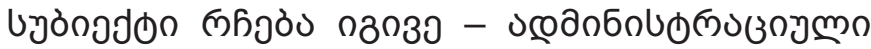

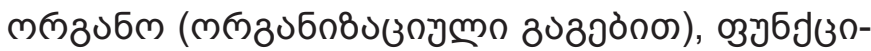

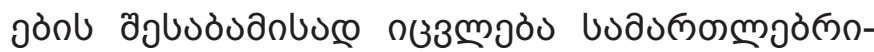

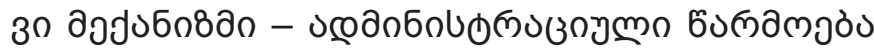

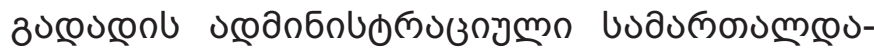

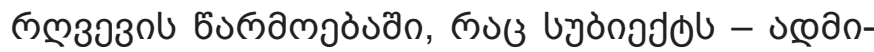

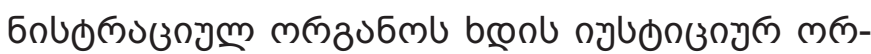

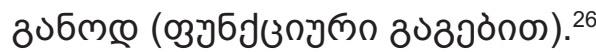

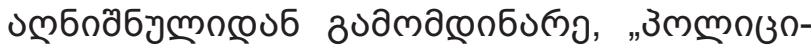

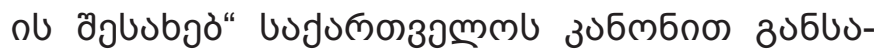

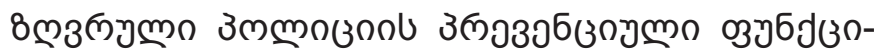

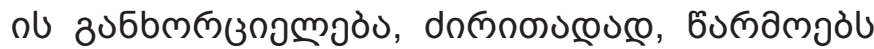

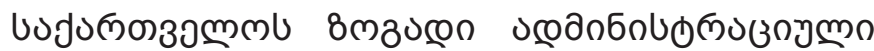

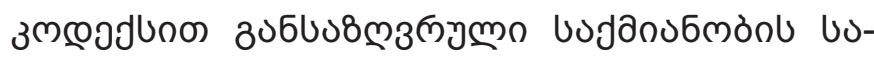

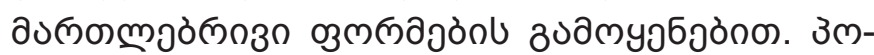

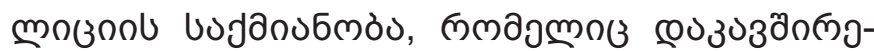

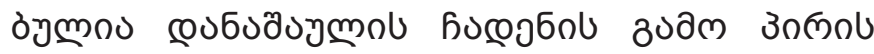

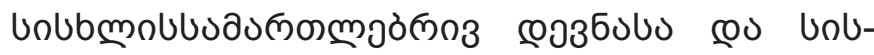

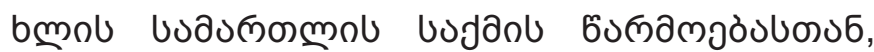

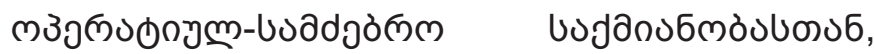

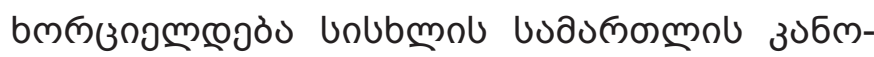

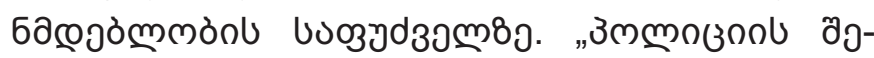

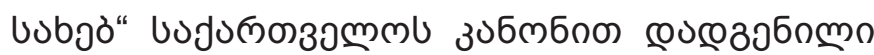

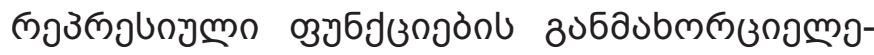

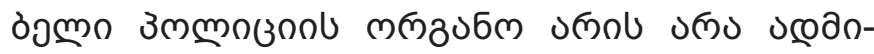

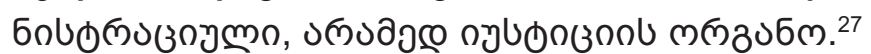

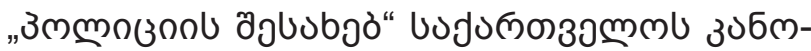

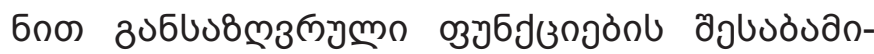

$1 \% 83 \% 98 \% \mathrm{E} 1 \% 83 \% \mathrm{~A} 1 \% \mathrm{E} 1 \% 83 \% 94 \% \mathrm{E} 1 \% 83 \% \mathrm{~A} 0$ $\%$ E $1 \% 83 \%$ A2\%E $1 \% 83 \% 90 \%$ E $1 \% 83 \%$ AA $\%$ E $1 \% 83$ $\% 98 \% \mathrm{E} 1 \% 83 \% 94 \% \mathrm{E} 1 \% 83 \% 91 \% \mathrm{E} 1 \% 83 \% 98 / \% \mathrm{E} 1 \%$ $83 \% 99 \%$ E $1 \% 83 \% 90 \%$ E $1 \% 83 \%$ AE $\%$ E $1 \% 83 \% 90 \% 20$ $\% \mathrm{E} 1 \% 83 \% \mathrm{~A} 7 \% \mathrm{E} 1 \% 83 \% \mathrm{~A} 3 \% \mathrm{E} 1 \% 83 \% \mathrm{~A} 0 \% \mathrm{E} 1 \% 83 \% 90$ $\% \mathrm{E} 1 \% 83 \% \mathrm{~A} 8 \% \mathrm{E} 1 \% 83 \% 95 \% \mathrm{E} 1 \% 83 \% 98 \% \mathrm{E} 1 \% 83 \% 9 \mathrm{~A}$ $\% \mathrm{E} 1 \% 83 \% 98$.pdf

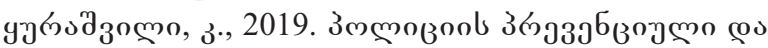

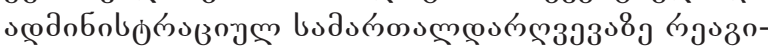

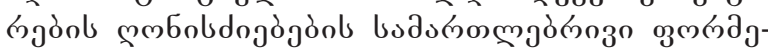

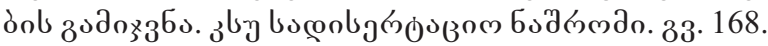
http://research.ciu.edu.ge/uploads/files/\%E1\%83\%93\%E $1 \% 83 \% 98 \% \mathrm{E} 1 \% 83 \% \mathrm{~A} 1 \% \mathrm{E} 1 \% 83 \% 94 \% \mathrm{E} 1 \% 83 \% \mathrm{~A} 0 \% \mathrm{E}$ $1 \% 83 \%$ A2\%E $\% 83 \% 90 \%$ E $1 \% 83 \%$ AA $\%$ E $1 \% 83 \% 98 \%$ E $1 \% 83 \% 94 \% \mathrm{E} 1 \% 83 \% 91 \% \mathrm{E} 1 \% 83 \% 98 / \% \mathrm{E} 1 \% 83 \% 99 \% \mathrm{E} 1$ $\% 83 \% 90 \% \mathrm{E} 1 \% 83 \%$ AE $\% \mathrm{E} 1 \% 83 \% 90 \% 20 \% \mathrm{E} 1 \% 83 \% \mathrm{~A} 7$ $\%$ E1\%83\%A3\%E1\%83\%A0\%E1\%83\%90\%E1\%83\%A8 $\% \mathrm{E} 1 \% 83 \% 95 \% \mathrm{E} 1 \% 83 \% 98 \% \mathrm{E} 1 \% 83 \% 9 \mathrm{~A} \% \mathrm{E} 1 \% 83 \% 98$. pdf

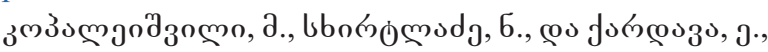

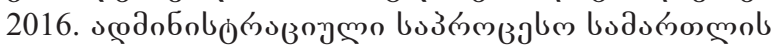

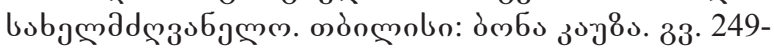
250 .

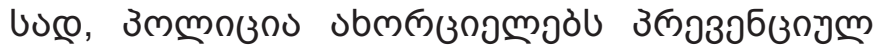

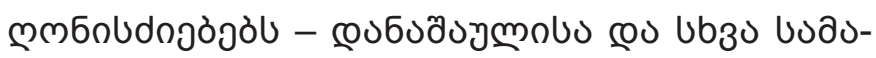

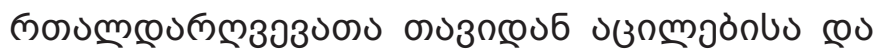

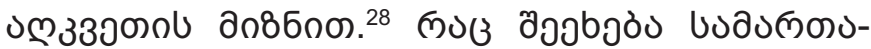

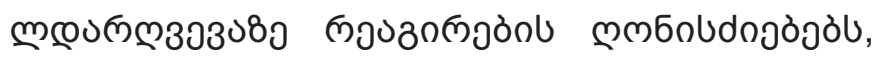

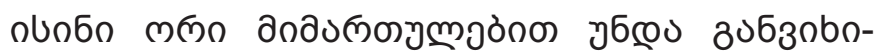

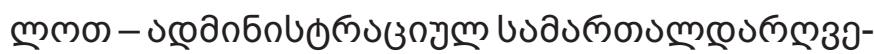

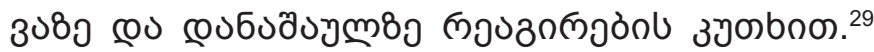

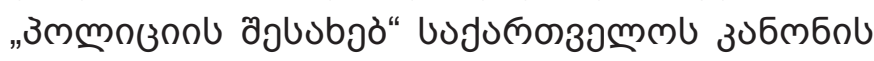

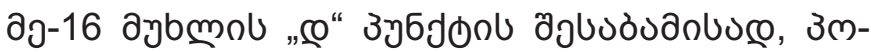

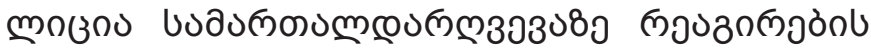

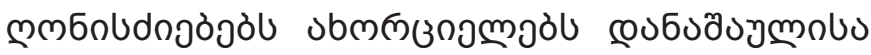

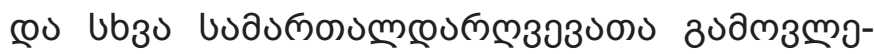

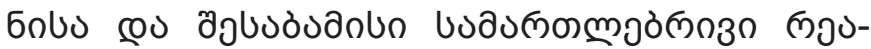

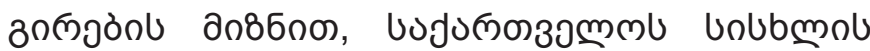

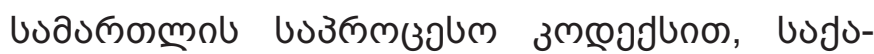

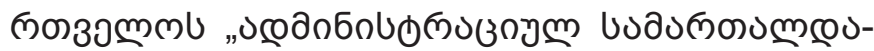

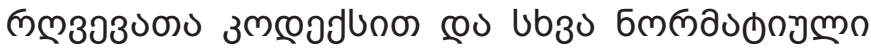

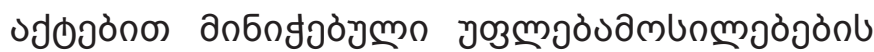

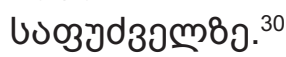

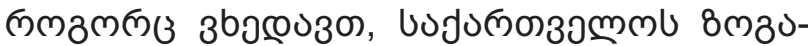

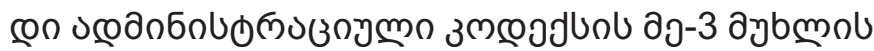

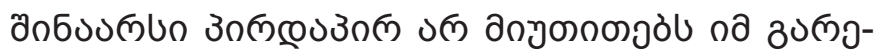

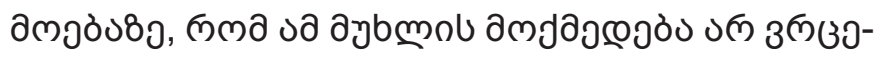

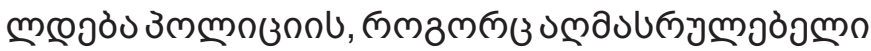

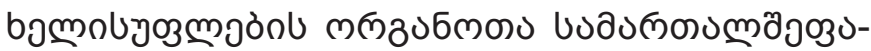

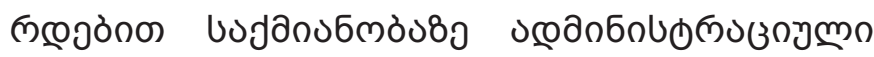

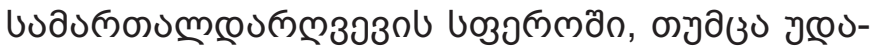

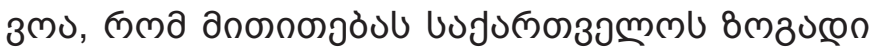

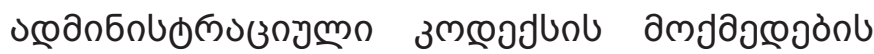

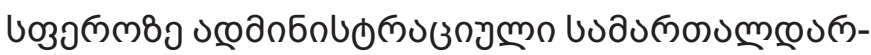

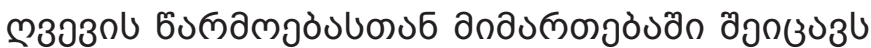

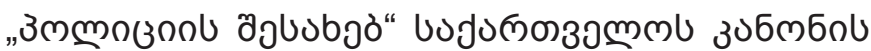
a-18 aybmol ao-2 6u6nmo cos aj-16 aybmol

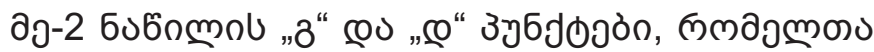

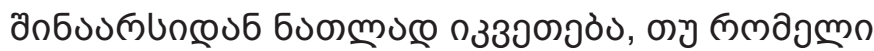

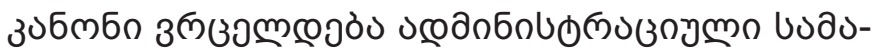

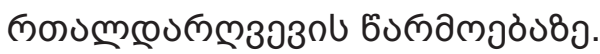

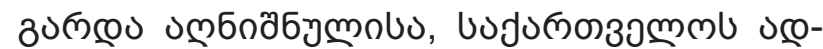

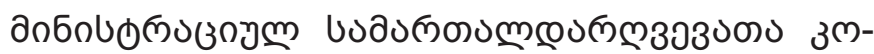
coodunu anboxoznm:

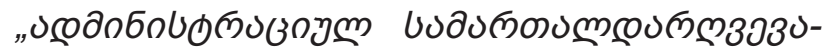

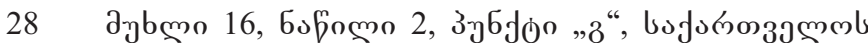
3 document/view/2047533?publication=29

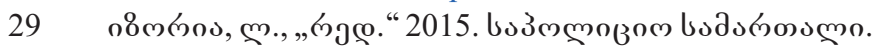

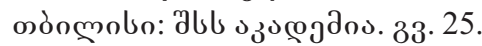

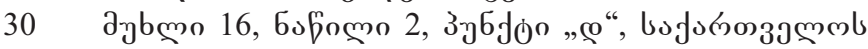

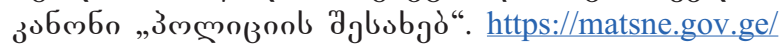
document/view/2047533?publication $=29$ 


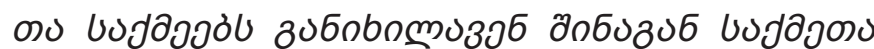

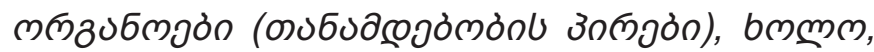

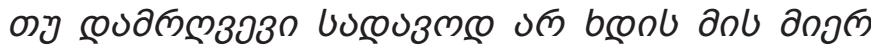

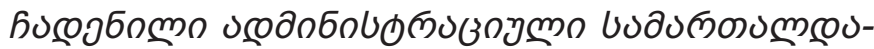

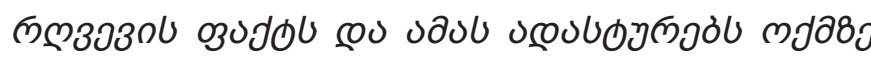

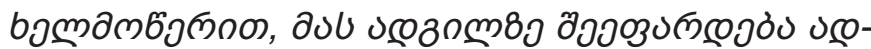

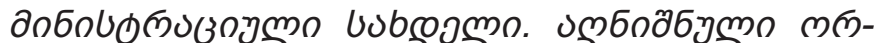

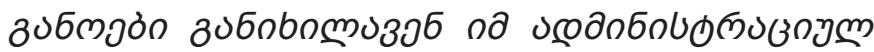

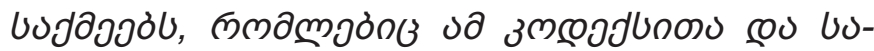

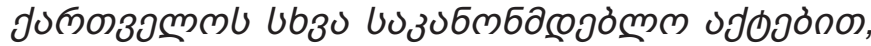

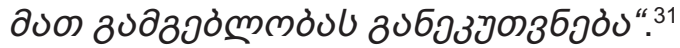

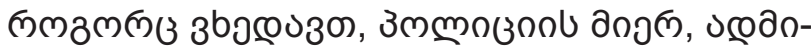

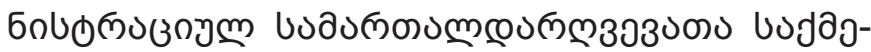

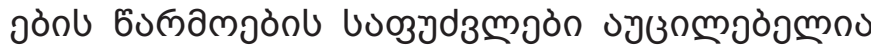

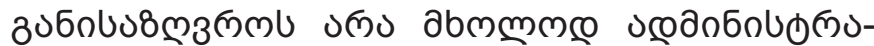

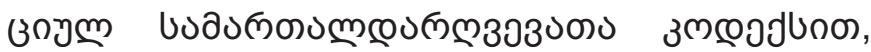

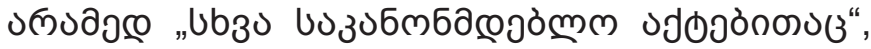

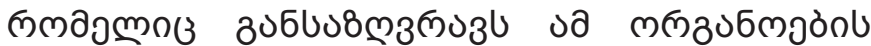

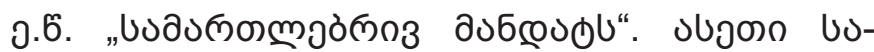

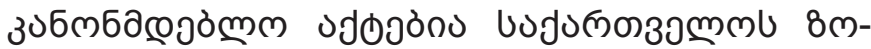

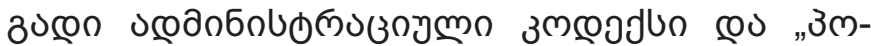

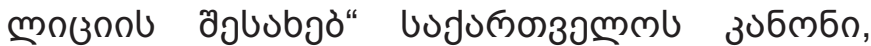

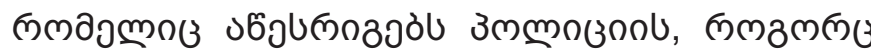

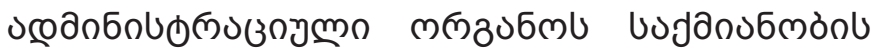

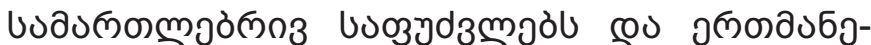

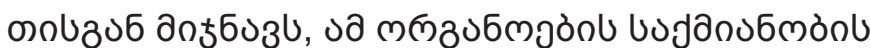

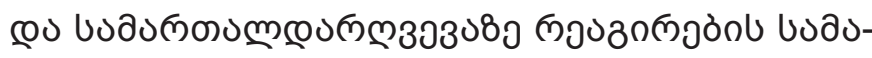

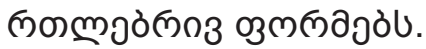

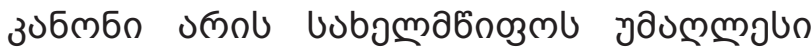

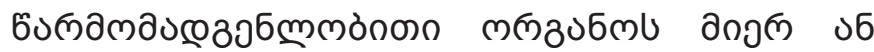

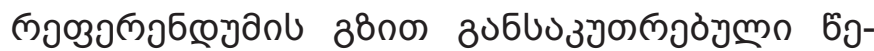

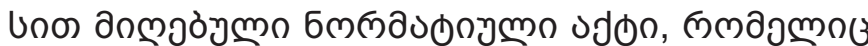

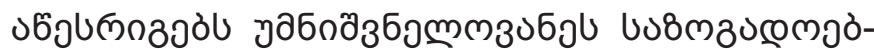

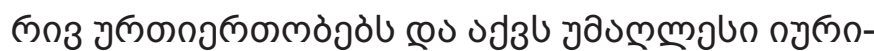

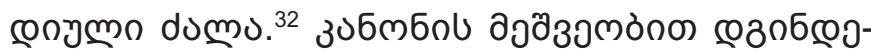

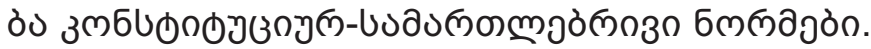

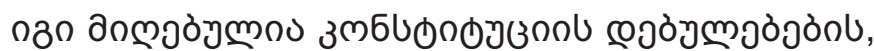

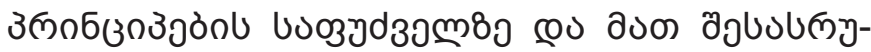
mó̀moro.

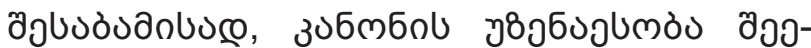

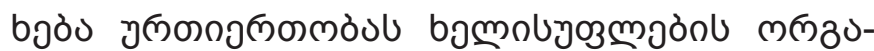

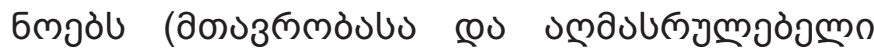

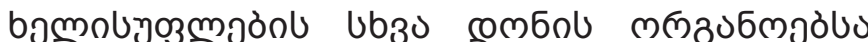

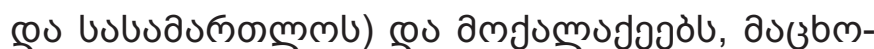

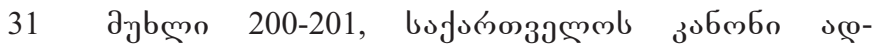

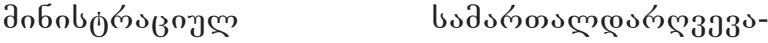
ол उмкоflo. https://matsne.gov.ge/document/ view $/ 28216$ ? publication $=476$

32 даб

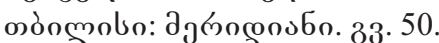

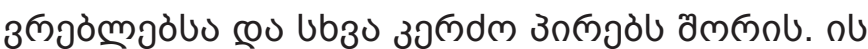

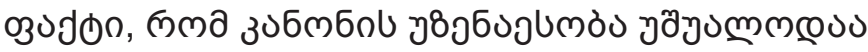

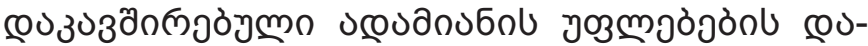

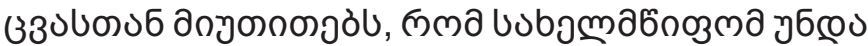

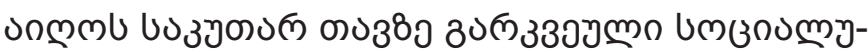

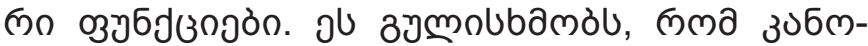

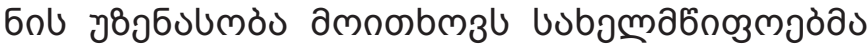

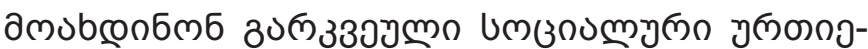

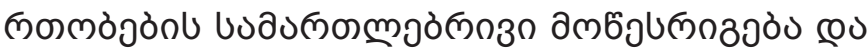

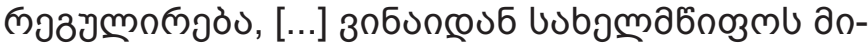

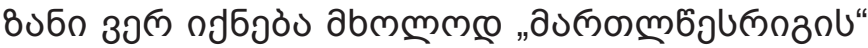

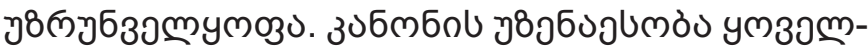

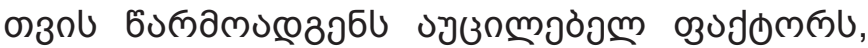

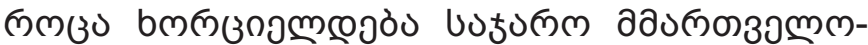

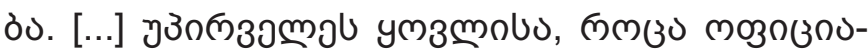

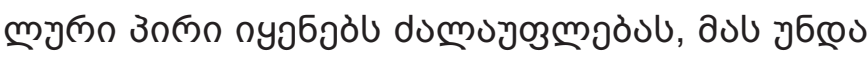

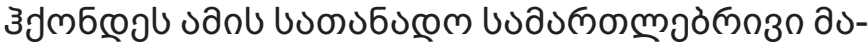

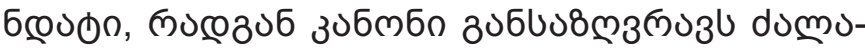

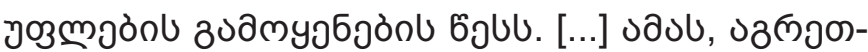

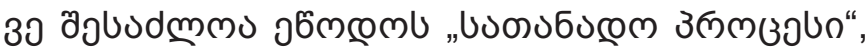

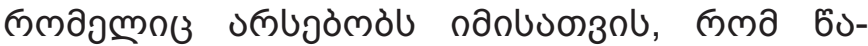

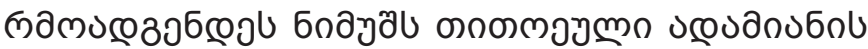

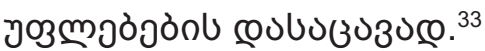

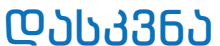

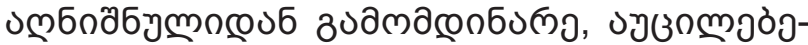

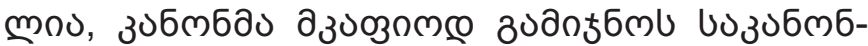

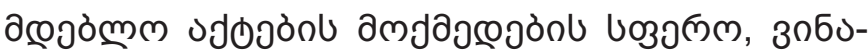

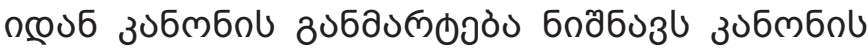

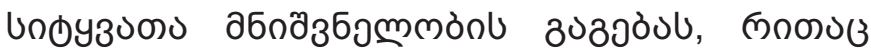

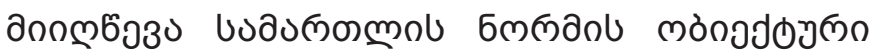

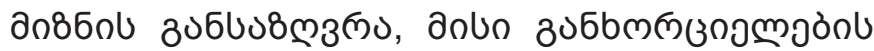

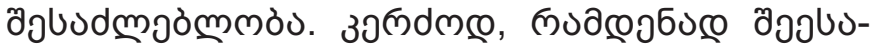

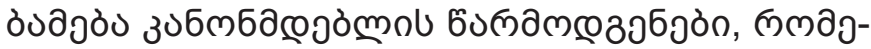

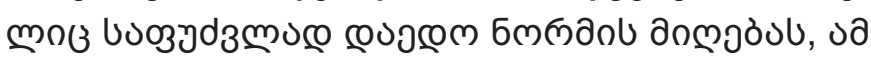

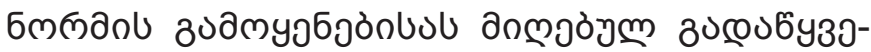

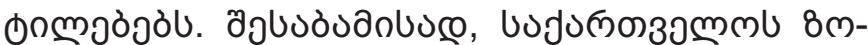

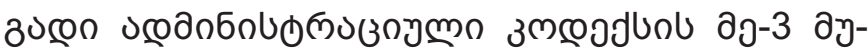

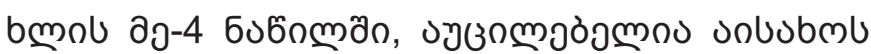

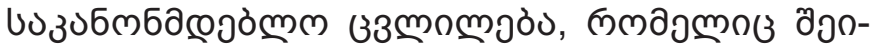

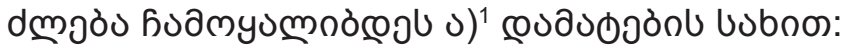

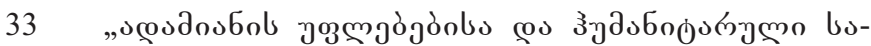

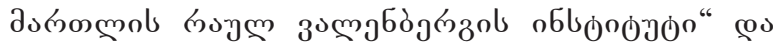

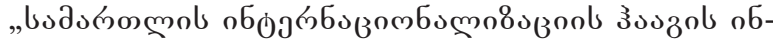

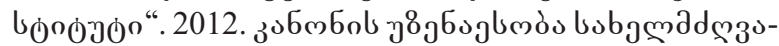

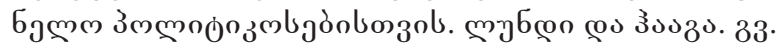
9-11. https://rwi.lu.se/app/uploads/2015/07/ruleoflawgeorgian.pdf 


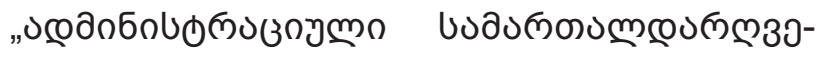

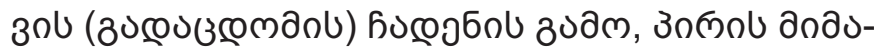

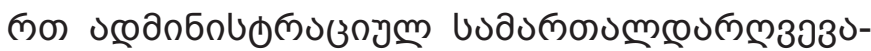

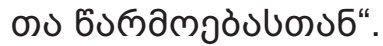

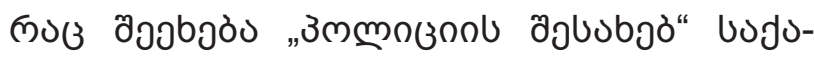

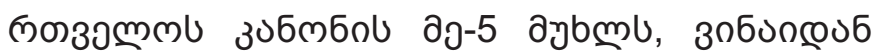

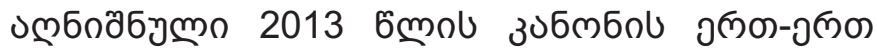

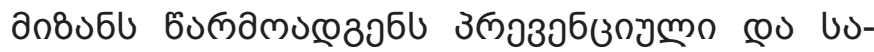

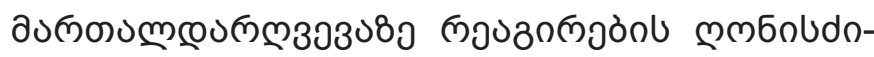

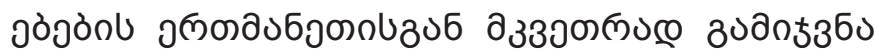

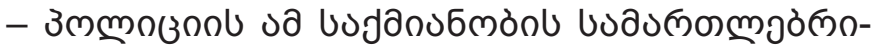

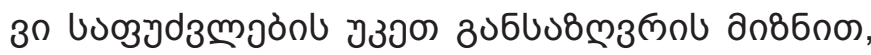

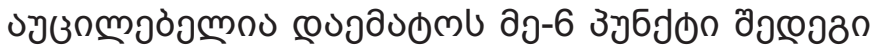

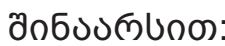

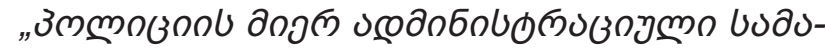

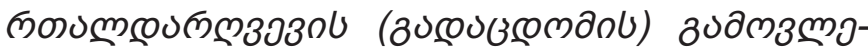

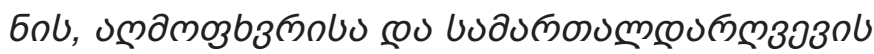

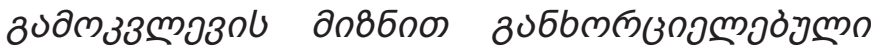

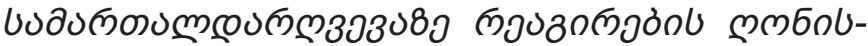

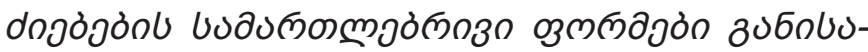

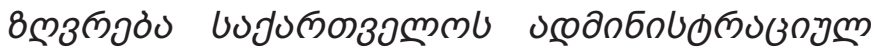

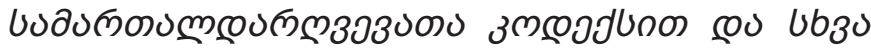

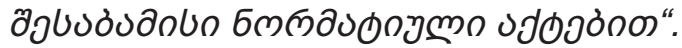

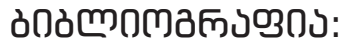

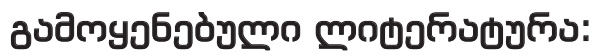

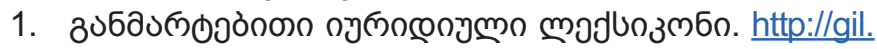
mylaw.ge/ka.html

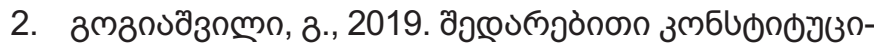

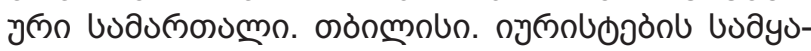
लm

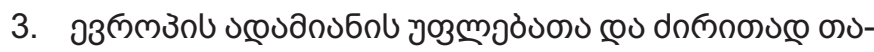

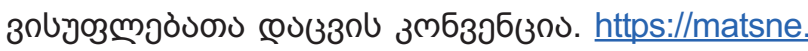
gov.ge/ka/document/view/1208370?publication $=0$

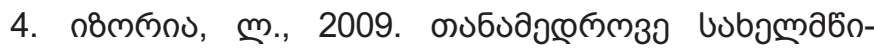

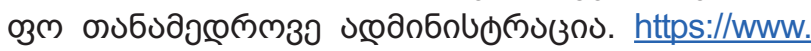
tsu.ge/data/file db/faculty-law-public/L[1].IzoriaTanamedrove\%20saxelmwifo.pdf

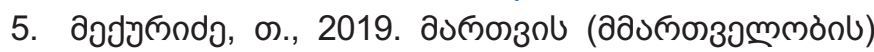

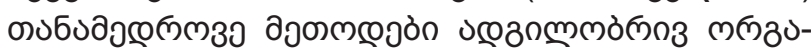

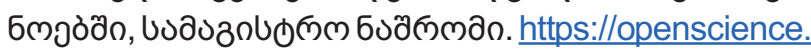
ge/bitstream/1/461/1/samagistro\%20mequridze.pdf

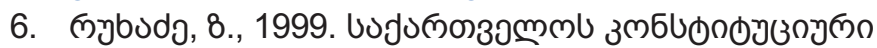

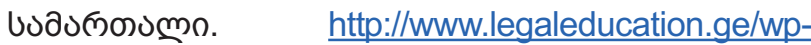
content/uploads/2011/03/gl 16 saqarTveloskonstituci urisamarTali.pdf

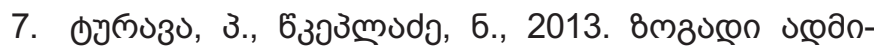

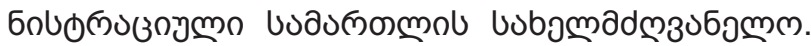

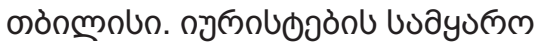

8. Wade, H.W.R., 1965. Administrative Law. Oxford

9. Maurer, H., 2011. Allgemeines Verwaltungsrecht. (18). Berlin

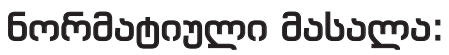

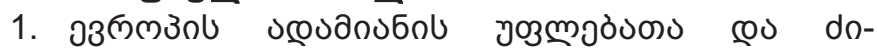

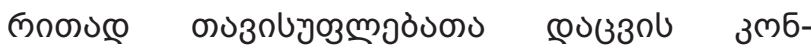
306300. $\quad$ https://matsne.gov.ge/ka/document/ view $/ 1208370$ ?publication $=0$

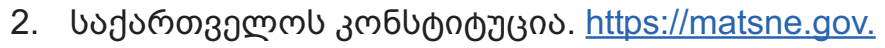

BIBLIOGRAPHY:

\section{Used Literature:}

1. Dictionary of Legal Terms. http://gil.mylaw.ge/ka.html (in Georgian)

2. Gogiashvili, G., 2019. Comparative Constitutional Law. Tbilisi: Publishing House World of Lawyers. (in Georgian)

3. European Convention on Human Rights and Freedoms. https://matsne.gov.ge/ka/document/ view/1208370?publication $=0$ (in Georgian)

4. Izoria, L., 2009. Modern State, Modern Administration. https://www.tsu.ge/data/file db/faculty-law-public/L[1].Izoria-Tanamedrove\%20saxelmwifo.pdf (in Georgian)

5. Mekuridze, T., 2019. Modern Methods of Management at Local Bodies, Master's thesis. https://openscience.ge/bitstream/1/461/1/samagistro\%20mequridze.pdf (in Georgian)

6. Rukhadze, Z., 1999. Constitutional Law of Georgia. http://www.legaleducation.ge/wp-content/uploads/2011/03/gl 16 saqarTveloskonstituciurisamarTali.pdf (in Georgian)

7. Turava, P., and Tskepladze, N., 2013. Guide to General Administrative Law. Tbilisi: Publishing House World of Lawyers. (in Georgian)

8. Wade, H.W.R., 1965. Administrative Law. Oxford. (in English)

9. Maurer, H., 2011. General Administrative Law. $18^{\text {th }}$ edition. Berlin. (in Germany)

\section{Normative material:}

1. European Convention on Human Rights and Freedoms. https://matsne.gov.ge/ka/document/ view $/ 1208370$ ?publication $=0$ (in Georgian)

2. Constitution of Georgia. https://matsne.gov.ge/ka/ document/view/30346?publication=36 (in Georgian) 
ge $/ \mathrm{ka} /$ document $/$ view $/ 30346$ ?publication $=36$

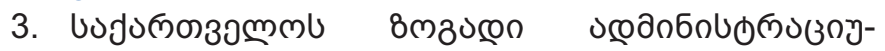
mo змкојЈ⿰丿. https://matsne.gov.ge/document/ view $/ 16270$ ?publication $=36$

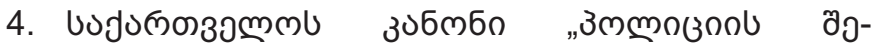
u৩bod". $\quad$ https://matsne.gov.ge/document/ view $/ 2047533$ ?publication $=29$

5. Бергманн, В. (2013). Административнопроцессуальное право Германии, Закон об Административном производстве Германии. (2-ое издание). Москва - Берлин: «infotropic media»
3. General Administrative Code of Georgia. https:// matsne.gov.ge/document/view/16270?publication=36 (in Georgian)

4. Law of Georgia „On Police“. https://matsne.gov.ge/ document/view/2047533?publication=29 (in Georgian)

5. Bergmann, V. 2013. Administrative Procedural Law of Germany, Law of Germany "Administrative Procedure Law". (second edition). Moscow-Berlin: Publishing House Infotropic media. (in Russian)

\section{NOTES:}

1. Zimmerman, cpt., Khubua, G., 2016. Legal Basics of Public Administration. Volume 3. Tbilisi: Publishing House SAMSHOBLO. p. 5, 62. (in Georgian)

2. Zimmerman, cpt., Khubua, G., 2016. Legal Basics of Public Administration. Volume 3. Tbilisi: Publishing House SAMSHOBLO. p. 63. (in Georgian)

3. Ibid, p. 18. (in Georgian)

4. Ibid, p. 107. (in Georgian)

5. Ibid, p. 108. (in Georgian)

6. Article 1, section 2, Law of Georgia General Administrative Code. https://matsne. gov.ge/document/view/16270?publication=36 (in Georgian)

7. Turava, P., 2018. General Administrative Law, second edition. Tbilisi: Publishing House World of Lawyers. p. 13-14, 16. (in Georgian)

8. Turava, P., 2016. General Administrative Law, second edition. Tbilisi: publishing house World of Lawyers. p. 12. Zimmerman, cpt., Khubua, G. 2016. Legal Basics of Public Administration. Volume 3. Tbilisi: Publishing House SAMSHOBLO. p. 32. (in Georgian)

9. Zimmerman, cpt., Khubua, G., 2016. Legal Basics of Public Administration. Volume 3. Tbilisi: Hublishing House SAMSHOBLO. p. 106. (in Georgian)

10. Kopaleishvili, M., Skhirtladze, N., and Kardava, E., 2016. Administrative Procedure Law Guide. Tbilisi: Publishing House BONA CAUSA. p. 33-34. (in Georgian)

11. Kurashvili, K., 2019. Separation of preventive and administrative offense respond legal forms of police. Dissertation Presented for the Degree of Doctor of Law. pp.64-65. http://research.ciu.edu.ge/uploads/files/\%E1\%83\%93\%E1\%83\%98\%E1 \%83\%A $1 \% \mathrm{E} 1 \% 83 \% 94 \% \mathrm{E} 1 \% 83 \% \mathrm{~A} 0 \% \mathrm{E} 1 \% 83 \% \mathrm{~A} 2 \% \mathrm{E} 1 \% 83 \% 90 \% \mathrm{E} 1 \% 83 \% \mathrm{AA} \%$ E1\%83\%98\%E1\%83\%94\%E1\%83\%91\%E1\%83\%98/\%E1\%83\%99\%E1\%83\%90 \%E1\%83\%AE\%E1\%83\%90\%20\%E1\%83\%A7\%E1\%83\%A3\%E1\%83\%A0\%E1\% 83\%90\%E1\%83\%A8\%E1\%83\%95\%E1\%83\%98\%E1\%83\%9A\%E1\%83\%98.pdf (in Georgian)

12. Loria, V. „Edit.“ 2004. Comments on Administrative Procedures Code of Georgia. Tbilisi: Publishing House MERIDIANI. p. 13. (in Georgian)

13. Kopaleishvili, M., Skhirtladze, N., and Kardava, E. 2016. Administrative Procedure Law Guide. Tbilisi: Publishing House BONA CAUSA. p. 36. (in Georgian)

14. Loria, V. 2002. Administrative Procedures Law of Georgia. Tbilisi: Publishing House MERIDIANI. p. 27. (in Georgian)

15. Kurashvili, K., 2019. Separation of preventive and administrative offense respond legal forms of police. Dissertation Presented for the Degree of Doctor of Law. $\mathrm{p}$. 66-67. http://research.ciu.edu.ge/uploads/files/\%E1\%83\%93\%E1\%83\%98\%E1\% 83\%A1\%E1\%83\%94\%E1\%83\%A0\%E1\%83\%A2\%E1\%83\%90\%E1\%83\%AA\%E 1\%83\%98\%E1\%83\%94\%E1\%83\%91\%E1\%83\%98/\%E1\%83\%99\%E1\%83\%90 \%E1\%83\%AE\%E1\%83\%90\%20\%E1\%83\%A7\%E1\%83\%A3\%E1\%83\%A0\%E1\% 83\%90\%E1\%83\%A8\%E1\%83\%95\%E1\%83\%98\%E1\%83\%9A\%E1\%83\%98.pdf 
(in Georgian)

16. Loria, V. 2002. Administrative Procedures Law of Georgia. Tbilisi: Publishing House MERIDIANI. p. 16. (in Georgian)

17. Kurashvili, K., 2019. Separation of preventive and administrative offense respond legal forms of police. Dissertation Presented for the Degree of Doctor of Law. p. 67-68. http://research.ciu.edu.ge/uploads/files/\%E1\%83\%93\%E1\%83\%98\%E1\% 83\%A1\%E1\%83\%94\%E1\%83\%A0\%E1\%83\%A2\%E1\%83\%90\%E1\%83\%AA\%E 1\%83\%98\%E1\%83\%94\%E1\%83\%91\%E1\%83\%98/\%E1\%83\%99\%E1\%83\%90 \%E1\%83\%AE\%E1\%83\%90\%20\%E1\%83\%A7\%E1\%83\%A3\%E1\%83\%A0\%E1\% 83\%90\%E1\%83\%A8\%E1\%83\%95\%E1\%83\%98\%E1\%83\%9A\%E1\%83\%98.pdf (in Georgian)

18. Banchuk, A., 2011. Administrative Law of Foreign Countries. Moscow: Walters Cluver. p. 62. (in Russian)

19. Ibid. p. 63. (in Russian)

20. Kurashvili, K., 2019. Separation of preventive and administrative offense respond legal forms of police. Dissertation Presented for the Degree of Doctor of Law. p. 69. http://research.ciu.edu.ge/uploads/files/\%E1\%83\%93\%E1\%83\%98\%E1\%83 \%A1\%E1\%83\%94\%E1\%83\%A0\%E1\%83\%A2\%E1\%83\%90\%E1\%83\%AA\%E1\% 83\%98\%E1\%83\%94\%E1\%83\%91\%E1\%83\%98/\%E1\%83\%99\%E1\%83\%90\%E 1\%83\%AE\%E1\%83\%90\%20\%E1\%83\%A7\%E1\%83\%A3\%E1\%83\%A0\%E1\%83 \%90\%E1\%83\%A8\%E1\%83\%95\%E1\%83\%98\%E1\%83\%9A\%E1\%83\%98.pdf (in Georgian)

21. Bergmann, V. 2013. Administrative Procedure Law of Germany, Law of Germany „On administrative proceedings“. (second edition). Moscow - Berlin: Publishing House Infotropic media. p. 9. (in Russian)

22. Kurashvili, K. 2019. Separation of preventive and administrative offense respond legal forms of police. Dissertation Presented for the Degree of Doctor of Law. $p$. 74-75. http://research.ciu.edu.ge/uploads/files/\%E1\%83\%93\%E1\%83\%98\%E1\% 83\%A1\%E1\%83\%94\%E1\%83\%A0\%E1\%83\%A2\%E1\%83\%90\%E1\%83\%AA\%E 1\%83\%98\%E1\%83\%94\%E1\%83\%91\%E1\%83\%98/\%E1\%83\%99\%E1\%83\%90 \%E1\%83\%AE\%E1\%83\%90\%20\%E1\%83\%A7\%E1\%83\%A3\%E1\%83\%A0\%E1\% 83\%90\%E1\%83\%A8\%E1\%83\%95\%E1\%83\%98\%E1\%83\%9A\%E1\%83\%98.pdf (in Georgian)

23. Turava, P. 2018. General Administrative Law. (second edition). Tbilisi: Publishing House World of Lawyers. p. 222. (in Georgian)

24. Kurashvili, K. 2019. Separation of preventive and administrative offense respond legal forms of police. Dissertation Presented for the Degree of Doctor of Law. p. 75. http://research.ciu.edu.ge/uploads/files/\%E1\%83\%93\%E1\%83\%98\%E1\%83 \%A1\%E1\%83\%94\%E1\%83\%A0\%E1\%83\%A2\%E1\%83\%90\%E1\%83\%AA\%E1\% 83\%98\%E1\%83\%94\%E1\%83\%91\%E1\%83\%98/\%E1\%83\%99\%E1\%83\%90\%E 1\%83\%AE\%E1\%83\%90\%20\%E1\%83\%A7\%E1\%83\%A3\%E1\%83\%A0\%E1\%83 \%90\%E1\%83\%A8\%E1\%83\%95\%E1\%83\%98\%E1\%83\%9A\%E1\%83\%98.pdf (in Georgian)

25. Kurashvili, K. 2019. Separation of preventive and administrative offense respond legal forms of police. Dissertation Presented for the Degree of Doctor of Law. p. 116. http://research.ciu.edu.ge/uploads/files/\%E1\%83\%93\%E1\%83\%98\%E1\%83 \%A1\%E1\%83\%94\%E1\%83\%A0\%E1\%83\%A2\%E1\%83\%90\%E1\%83\%AA\%E1\% 83\%98\%E1\%83\%94\%E1\%83\%91\%E1\%83\%98/\%E1\%83\%99\%E1\%83\%90\%E 1\%83\%AE\%E1\%83\%90\%20\%E1\%83\%A7\%E1\%83\%A3\%E1\%83\%A0\%E1\%83 \%90\%E1\%83\%A8\%E1\%83\%95\%E1\%83\%98\%E1\%83\%9A\%E1\%83\%98.pdf (in Georgian)

26. Kurashvili, K., 2019. Separation of preventive and administrative offense respond legal forms of police. Dissertation Presented for the Degree of Doctor of Law. p. 168. http://research.ciu.edu.ge/uploads/files/\%E1\%83\%93\%E1\%83\%98\%E1\%83 \%A1\%E1\%83\%94\%E1\%83\%A0\%E1\%83\%A2\%E1\%83\%90\%E1\%83\%AA\%E1\% 83\%98\%E1\%83\%94\%E1\%83\%91\%E1\%83\%98/\%E1\%83\%99\%E1\%83\%90\%E 1\%83\%AE \%E1\%83\%90\%20\%E1\%83\%A7\%E1\%83\%A3\%E1\%83\%A0\%E1\%83 

Georgian)

27. Kopaleishvili, M., Skhirtladze, N., and Kardava, E., 2016. Administrative Procedure Law Guide. Tbilisi: Publishing House BONA CAUSA. p. 249-250. (in Georgian)

28. Article 16, part 2, Section "G", Law of Georgia "On Police". https://matsne.gov.ge/ document/view/2047533?publication=29 (in Georgian)

29. Izoria, L. „Edit“. 2015. Police Law. Tbilisi: MIA Academy publishing. p. 25. (in Georgian)

30. Article 16, part 2, Section "D", Law of Georgia "On Police". https://matsne.gov.ge/ document/view/2047533?publication=29 (in Georgian)

31. Articles 200-201, Law of Georgia Administrative Offense Code. https://matsne.gov. ge/document/view/28216?publication $=476$ (in Georgian)

32. Shengelia, R. „Edit.“ 2003. Jurisprudence. Tbilisi: Publishing House MERIDIANI. p. 50. (in Georgian)

33. "Raoul Wallenberg Institute of Human Rights and Humanitarian Law" and "The Hague Institute for the Internationalization of Law". 2012. "Rule of Law: A Guide for Politicians". Lund and Hague. p. 9-11. https://rwi.lu.se/app/uploads/2015/07/ ruleoflaw-georgian.pdf (in Georgian) 